\title{
Ozonation of cylindrospermopsin (cyanotoxin): degradation mechanisms and cytotoxicity assessments
}

\author{
Shuwen Yan ${ }^{1}$, Ai Jia ${ }^{2}$, Sylvain Merel ${ }^{2}$, Shane A Snyder ${ }^{2,}$, Kevin E. O'Shea ${ }^{3}$, Dionysios D. Dionysiou ${ }^{4}$ \\ and Weihua Song ${ }^{1, *}$ \\ ${ }^{1}$ Department of Environmental Science \& Engineering, Fudan University, Shanghai, 200433, China \\ ${ }^{2}$ Department of Chemical \& Environmental Engineering, University of Arizona, Tucson, AZ, 85721, \\ US \\ ${ }^{3}$ Department of Chemistry \& Biochemistry, Florida International University, Miami, FL, 33199, US \\ ${ }^{4}$ Environmental Engineering \& Science Program, University of Cincinnati, Cincinnati, OH, 45221, US
}

Prepared for Environ. Sci. \& Technol.

Corresponding authors:

Shane A. Snyder email: snyders2@email.arizona.edu Tel:(+1) 5206212573 Weihua Song email: wsong@,fudan.edu.cn Tel: $(+86) 2165642040$

33 pages

1 Table (S1)

6 Figures (S1-S6) 
Table S1 The detailed mobile phase gradient for the acquisition method

\begin{tabular}{ccc}
\hline \hline Time (mins) & $\begin{array}{c}\text { Water }+\begin{array}{c}0.1 \% \text { formic acid } \\
(\%)\end{array} \\
0\end{array}$ & $\begin{array}{c}\text { Acetonitrile }+0.1 \% \text { formic } \\
\text { acid }\end{array}$ \\
\hline 5 & 98 & 2 \\
8 & 98 & 2 \\
10 & 60 & 40 \\
12 & 60 & 40 \\
12.01 & 40 & 60 \\
15.01 & 10 & 90 \\
& 10 & 90 \\
\hline
\end{tabular}




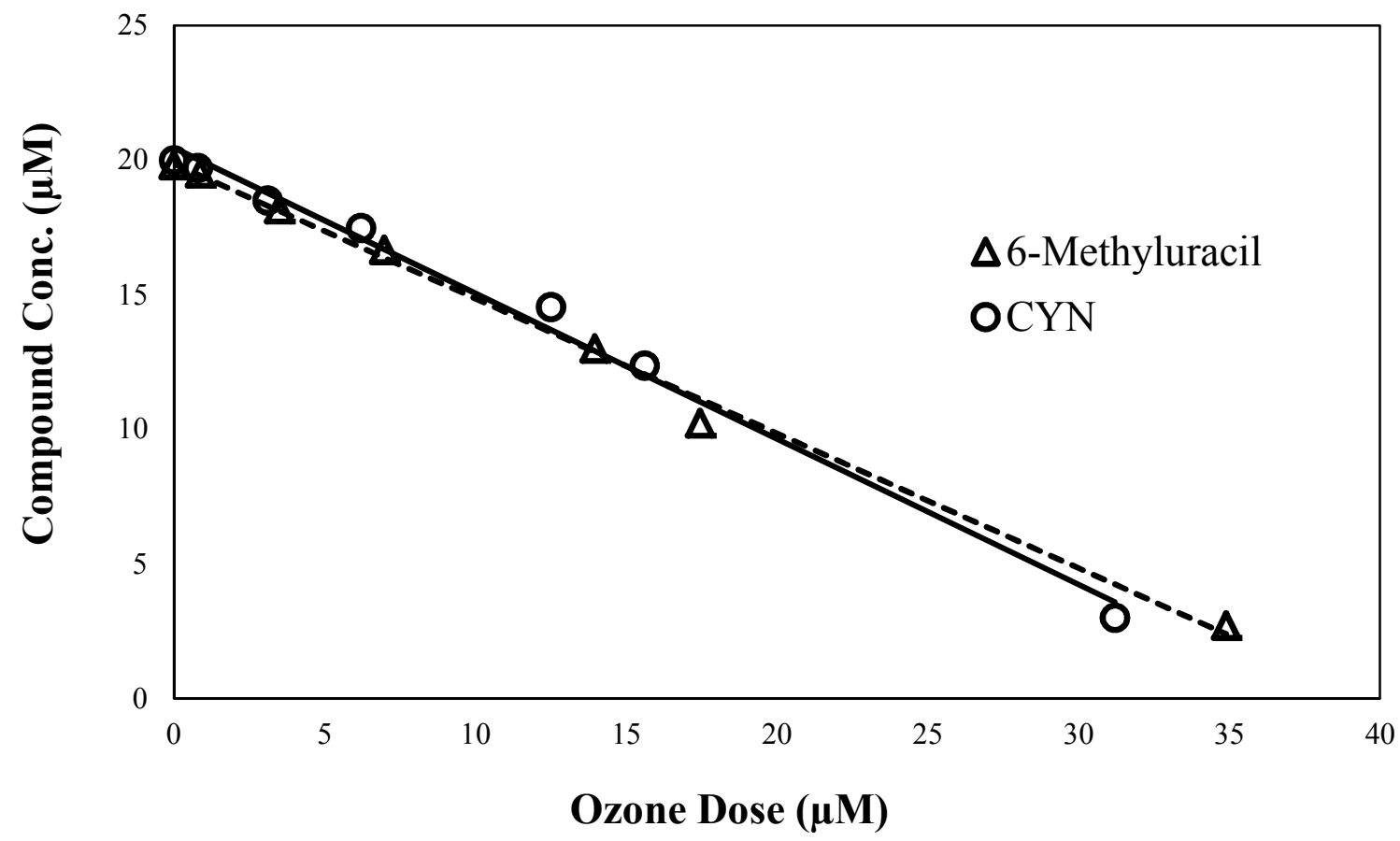

Figure S1.Concentrations of CYN and 6-methyluracil in distilled water as a function of ozone doses. Initial concentrations of CYN and 6-methyluracil were $20.0 \mu \mathrm{M}$. 

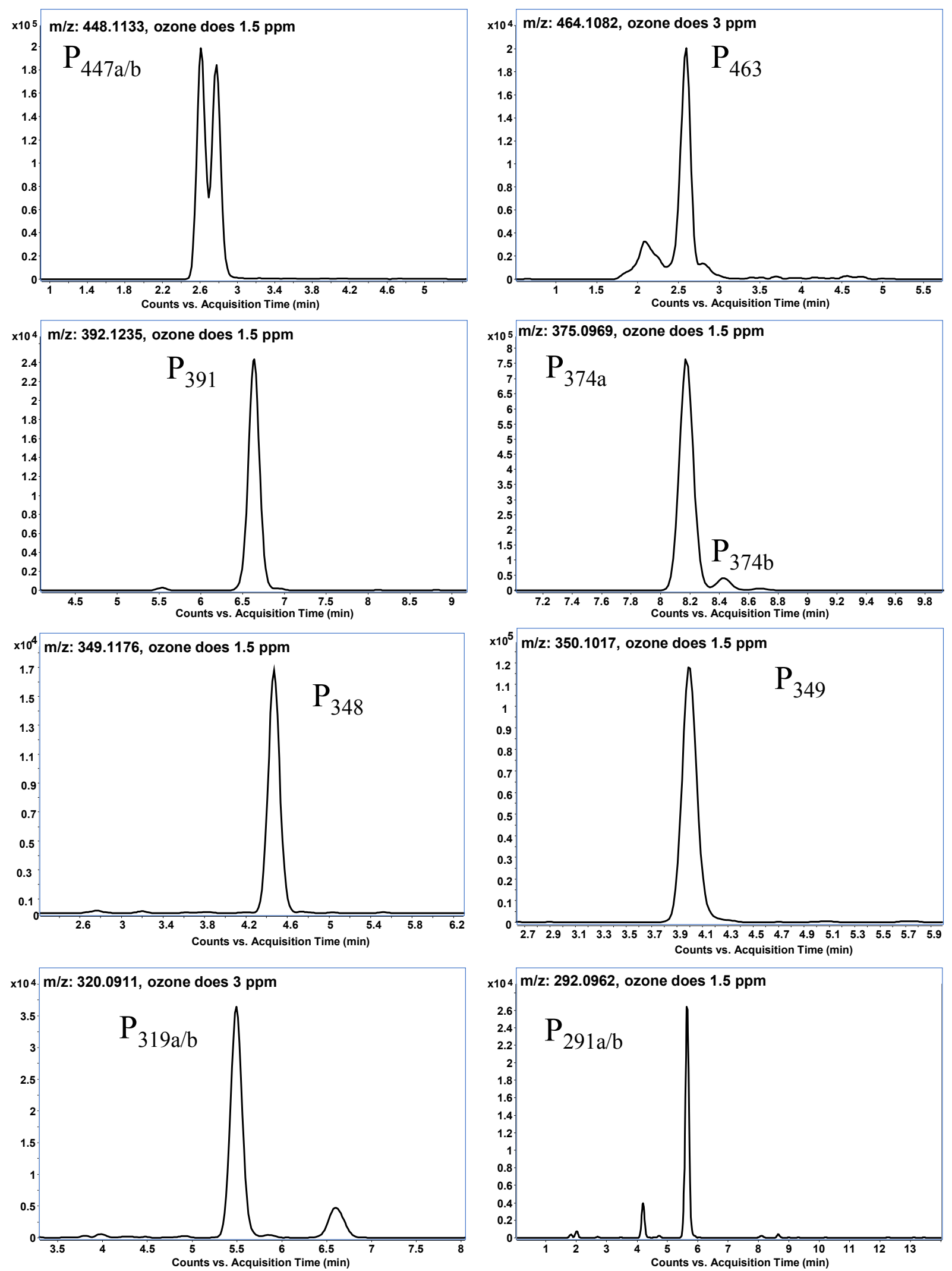

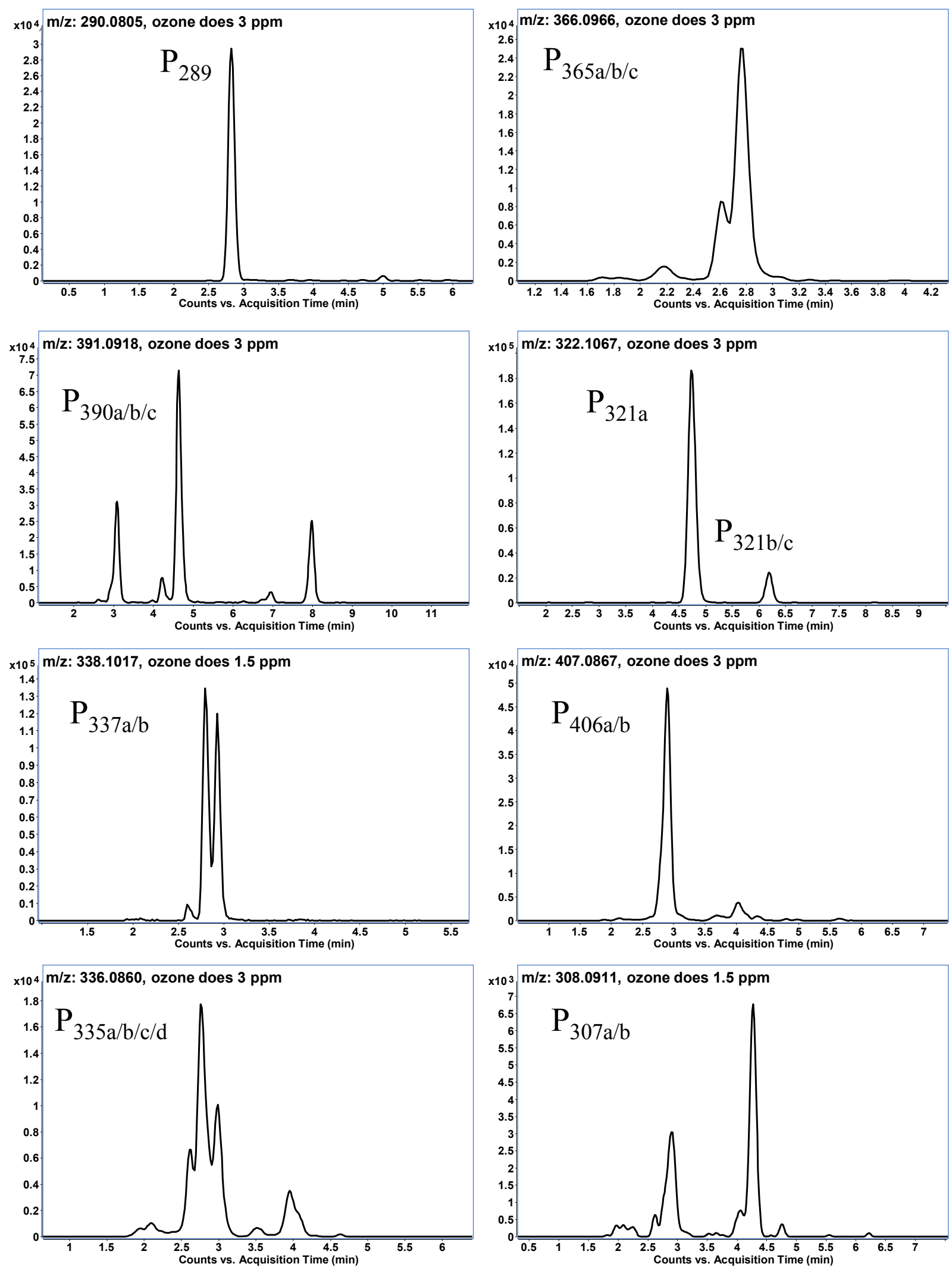


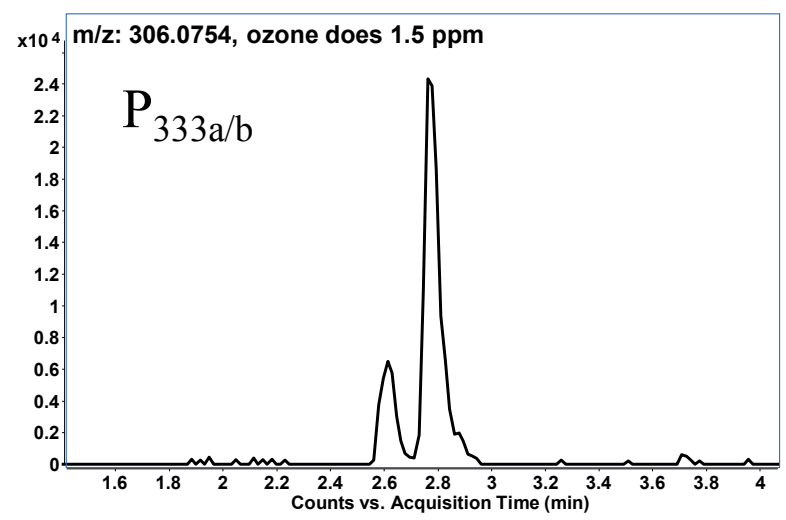

Figure S2. The extracted-ion mass chromatographs for the ozonation products of CYN. All figures presented the highest intensity of each product at the corresponding ozone dose. 


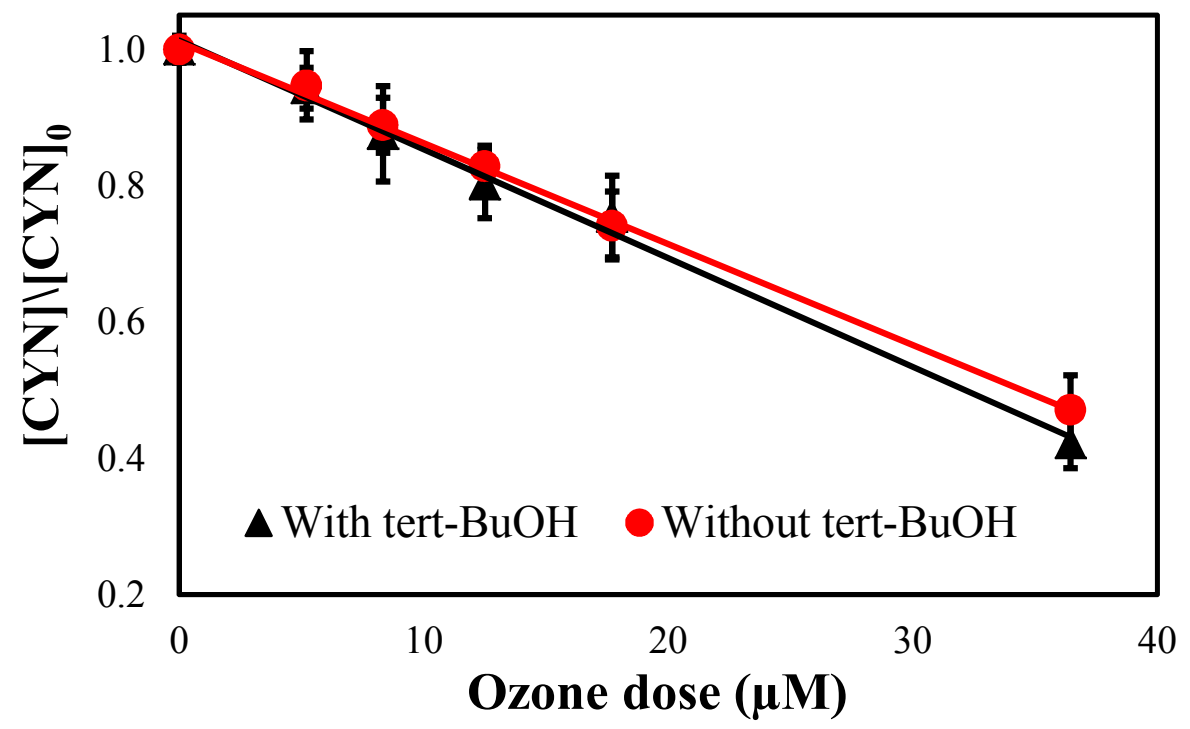

Figure S3. Degradation of CYN with or without tert-BuOH at various ozone doses. 
$\mathrm{m} / \mathrm{z}=416$

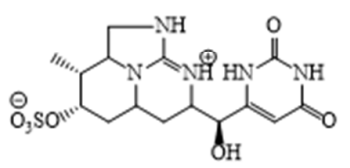

m/z: 416.124

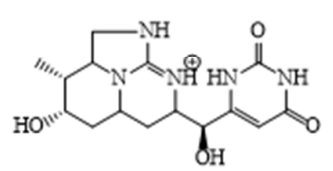

m/2: 336.167

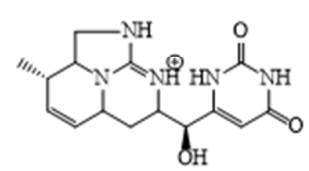

m/2: 318.156
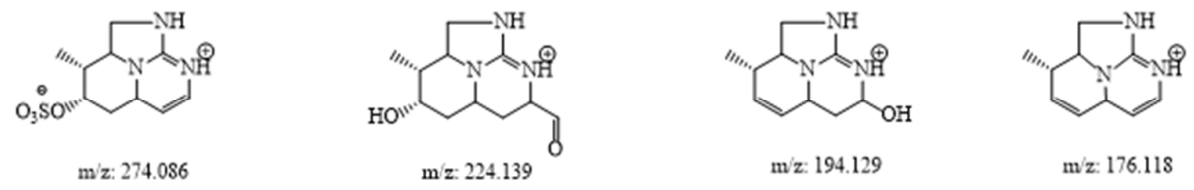

m/z: 194.129

m/z: 176.118

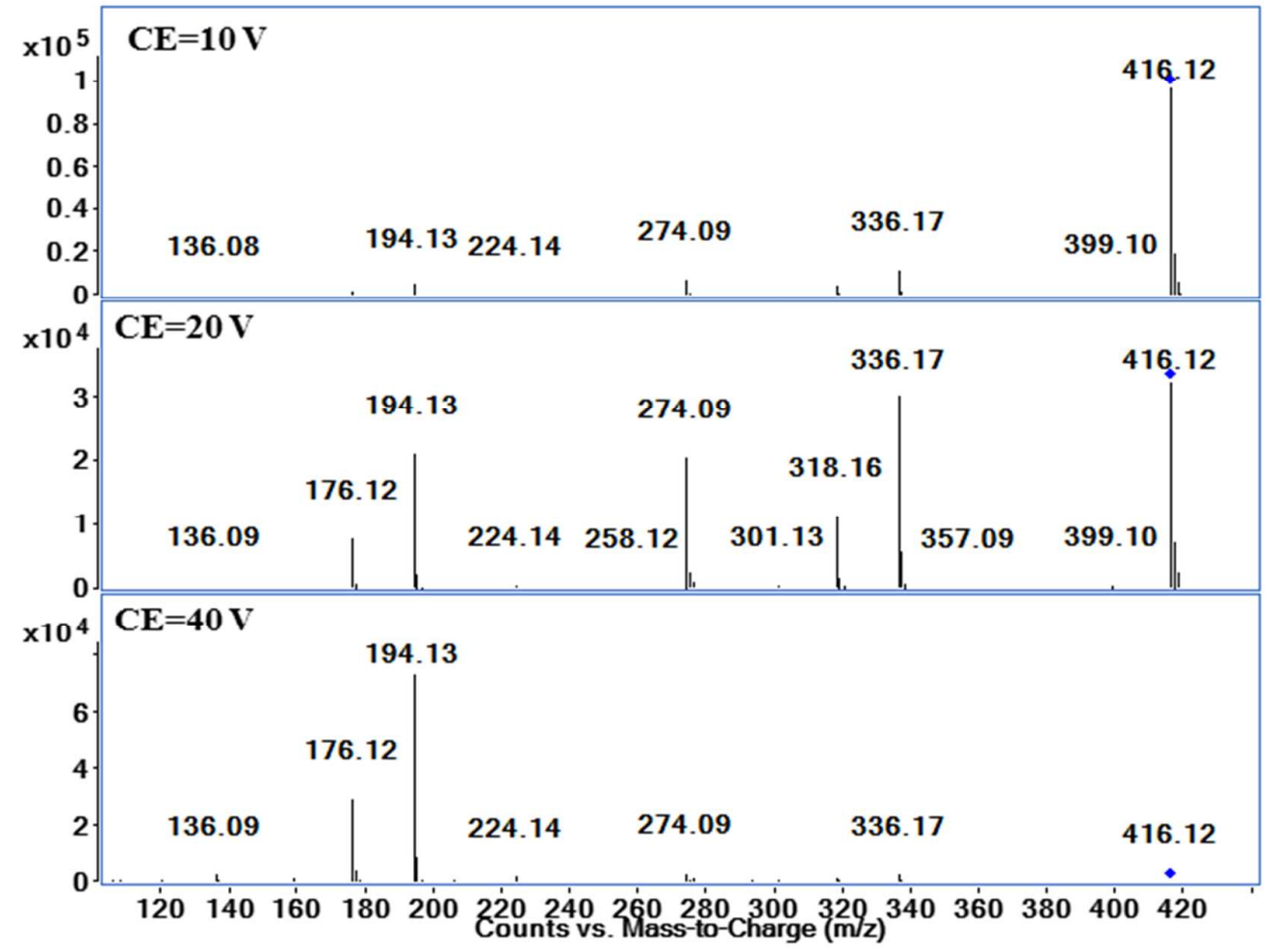




\section{$P_{447 a}$}

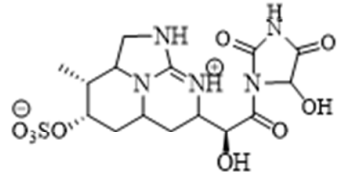

m/z: 448.11

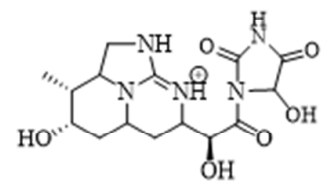

m/z: 368.16

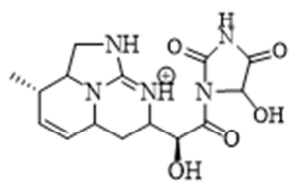

m/z: 350.15

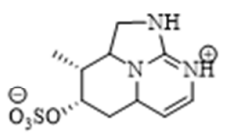

$\mathrm{m} / \mathrm{z}: 274.086$
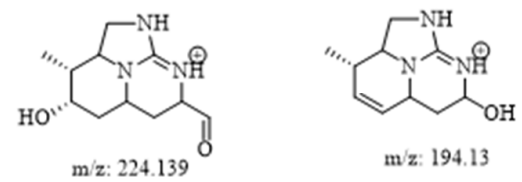

m/2: 194.13

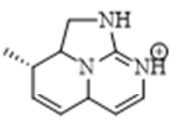

m/z: 176.12

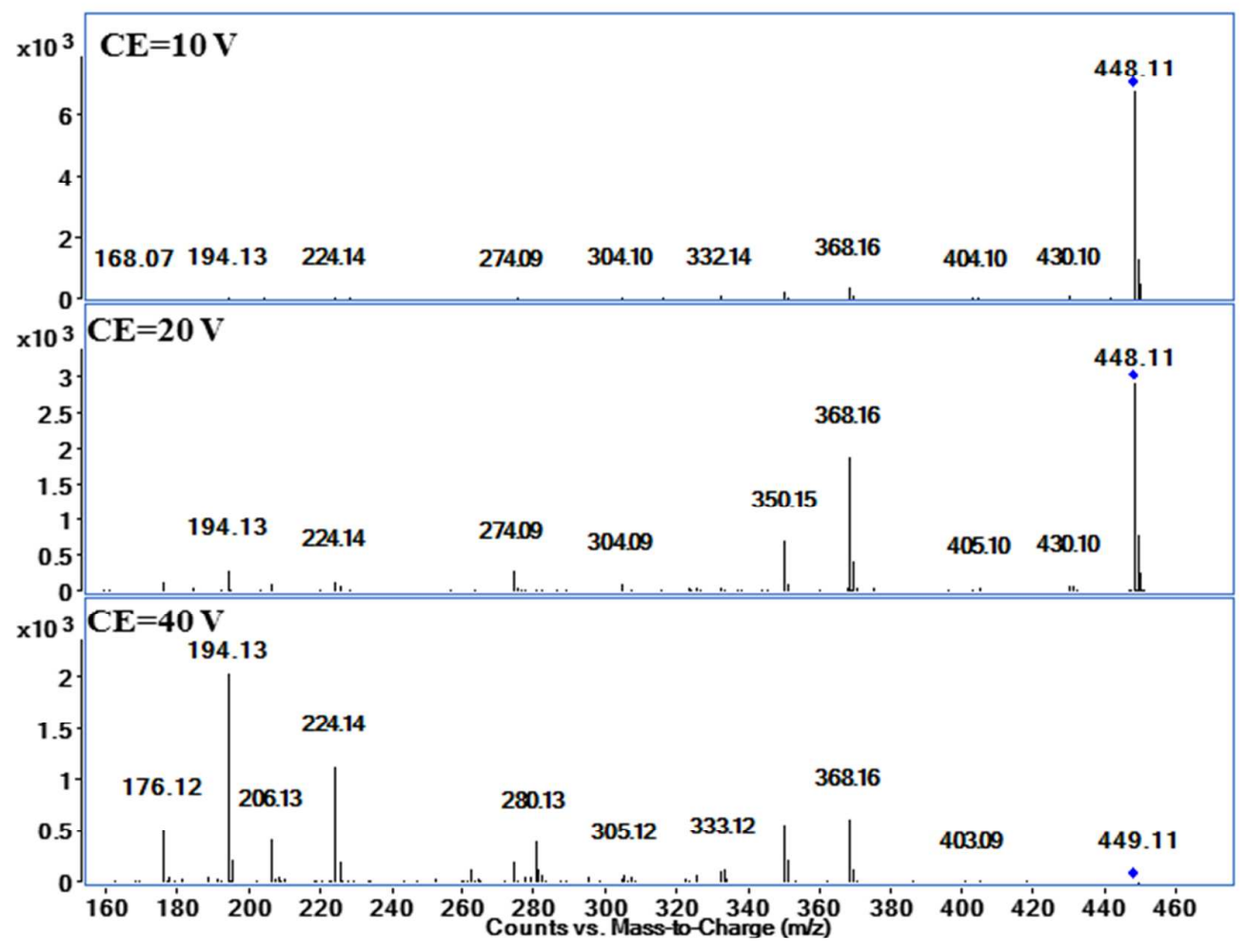




\section{$\mathbf{P}_{447 \mathrm{~b}}$}
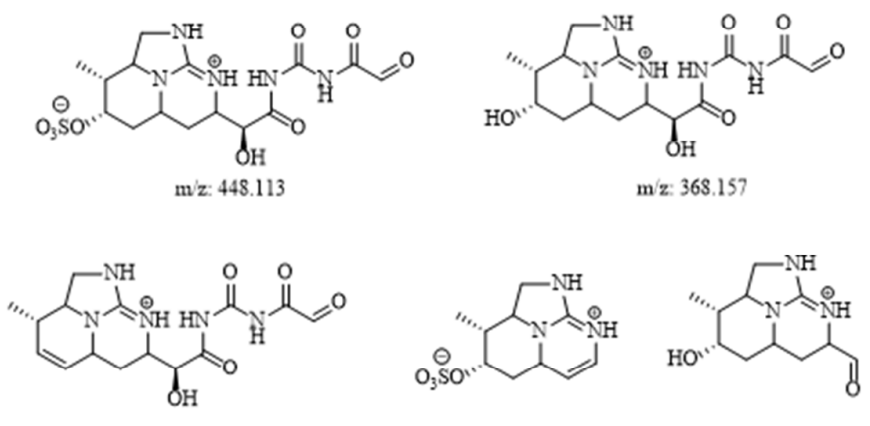

m/z: 350.146

$m / z=274.086$

m/z: 224.139
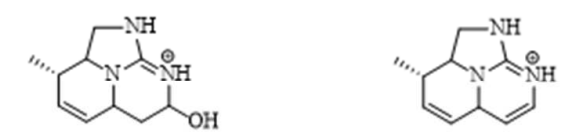

m/z: 194.13

miz: 176.12

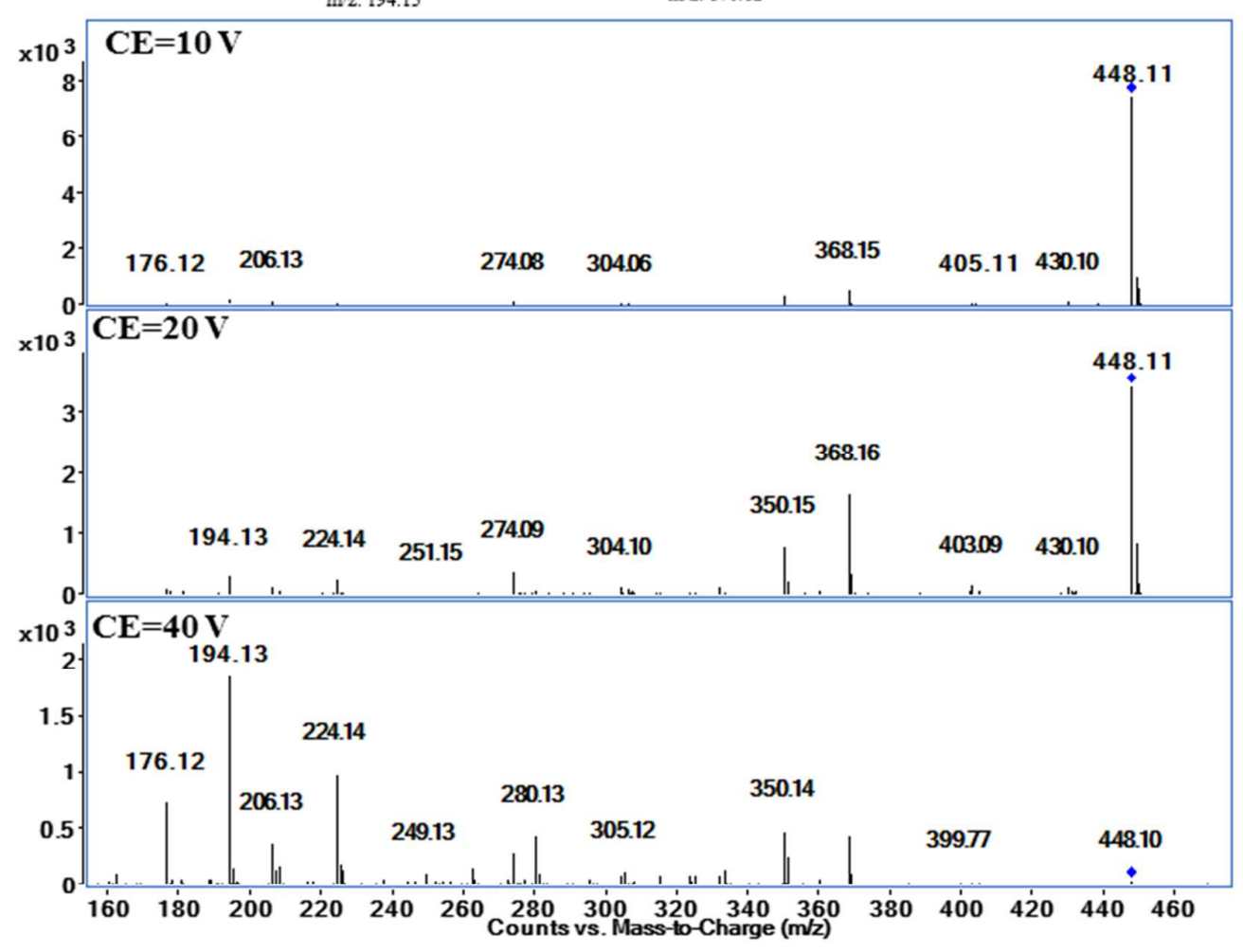




\section{$\mathbf{P}_{391}$}

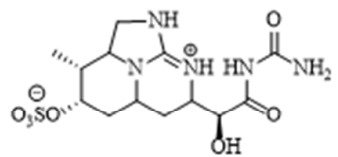

m/z: 392.123

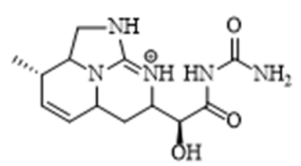

$\mathrm{m} / \mathrm{z}=294.156$

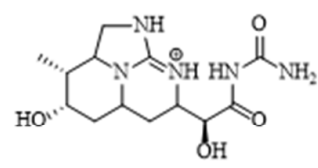

m/z: 312.167

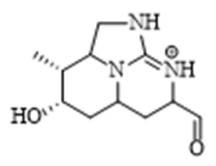

m/z: 224.139

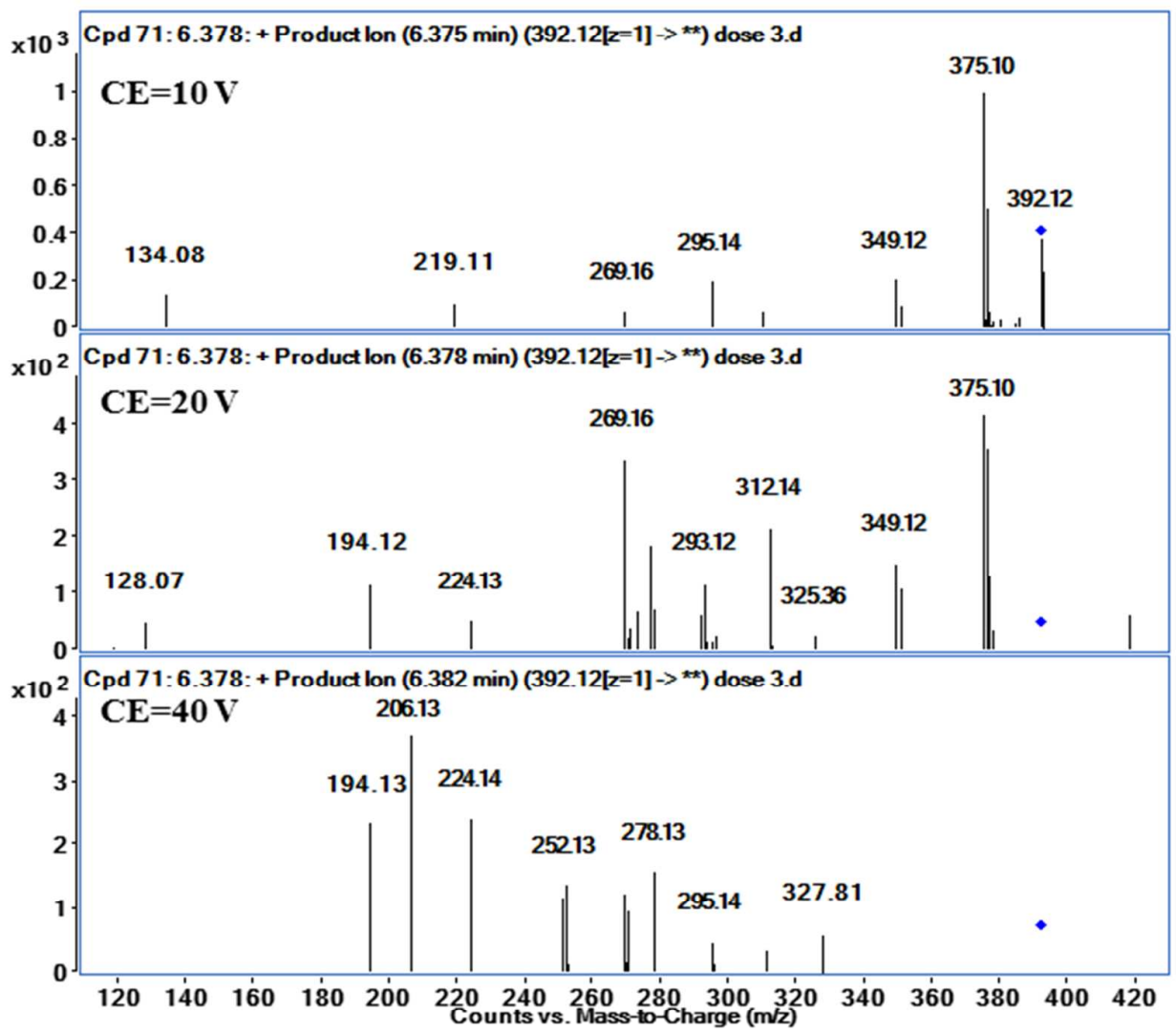




\section{$\mathbf{P}_{374 a}$}

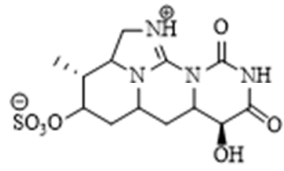

m/z: 375.097

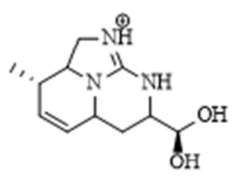

m/z: 224.139

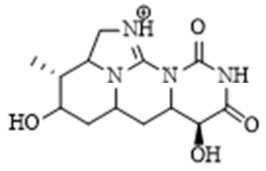

m/2: 295.140

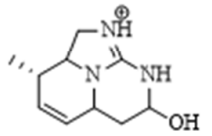

$\mathrm{m} / \mathrm{z}: 194.129$

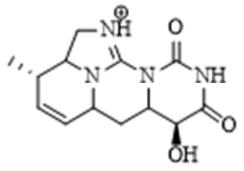

m/z: 277.130

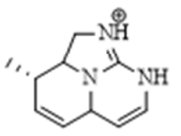

$\mathrm{m} / \mathrm{z}: 176.118$

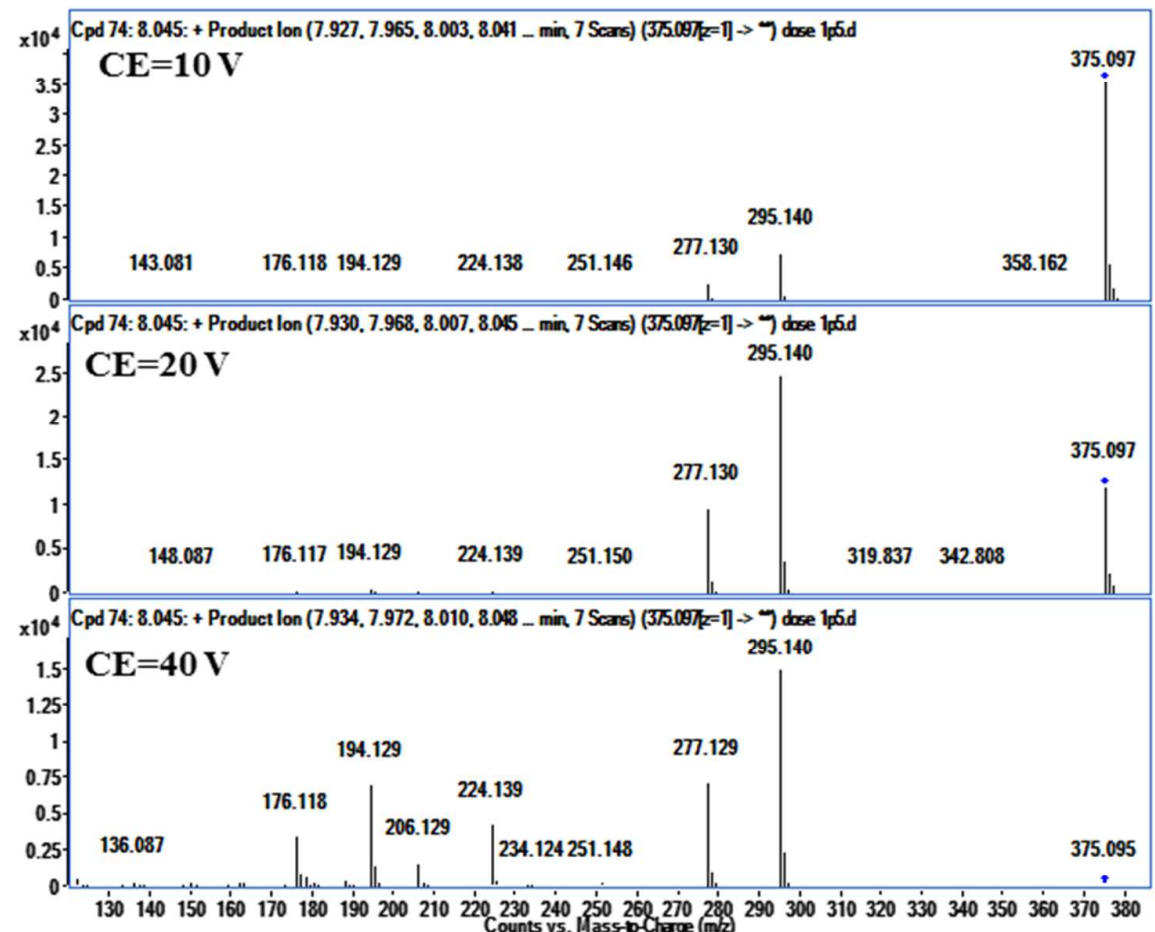

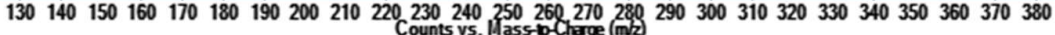




\section{$\mathbf{P}_{\text {374b }}$}

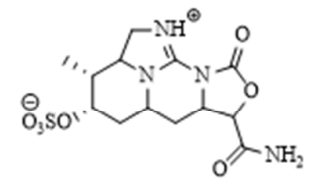

m/z: 375.097

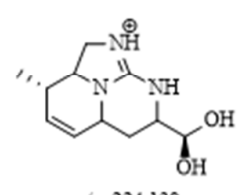

$\mathrm{m} / \mathrm{z}=224.139$

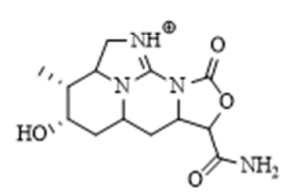

$\mathrm{m} / \mathrm{z}: 295.140$

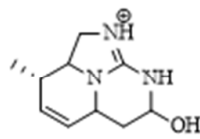

m/z: 194.129

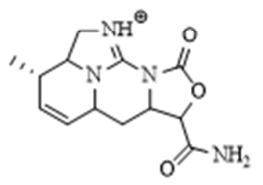

m/z: 277.130

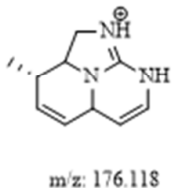

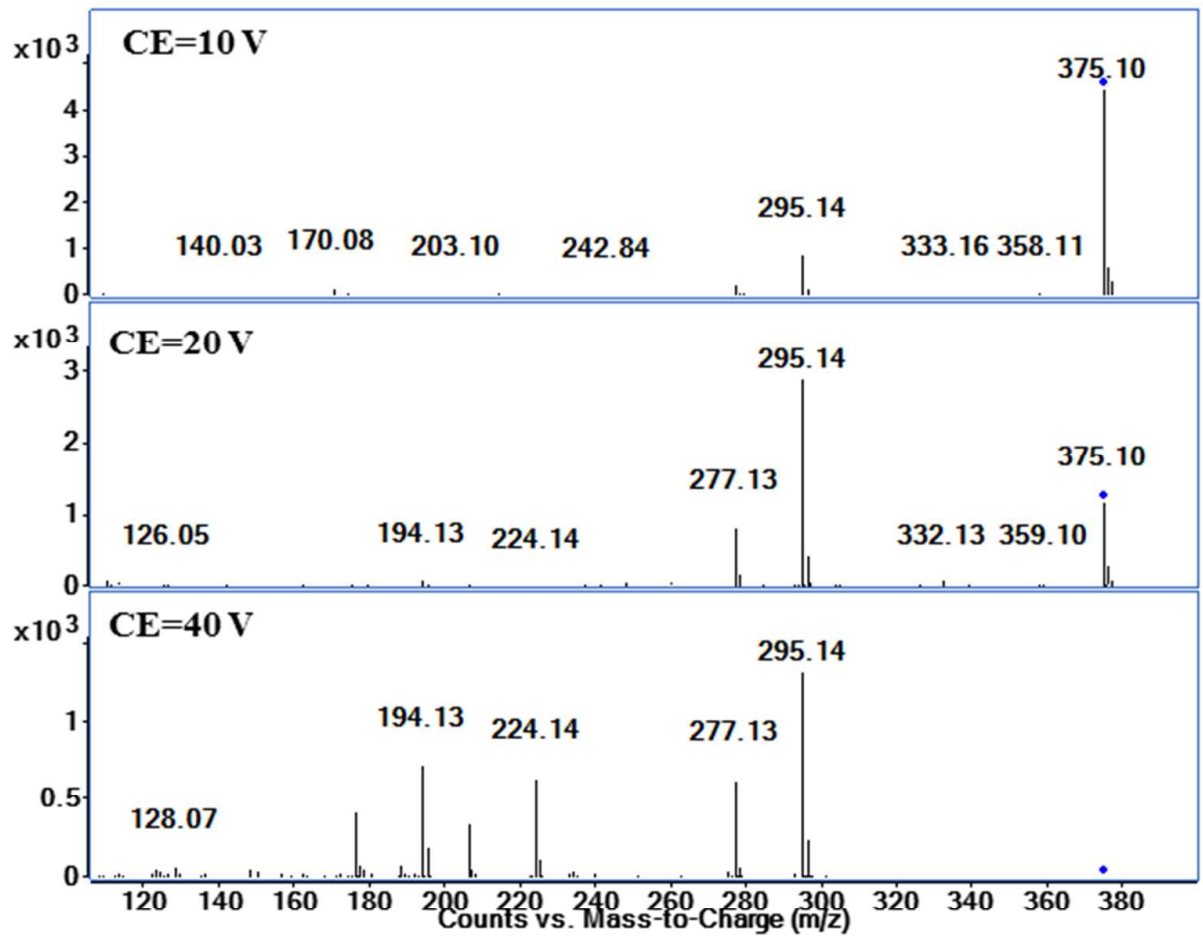




\section{$\mathbf{P}_{348}$}

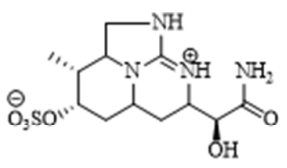

m/z: 349.12

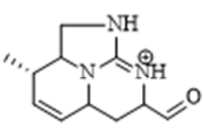

m/z: 206.13

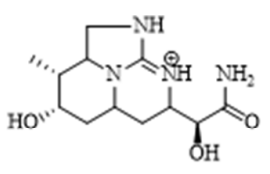

m/z: 269.16

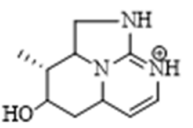

m/z: 194.13

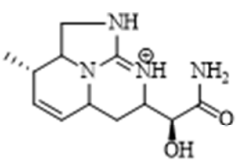

m/z: 251.15

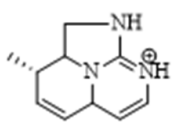

m/z: 176.12

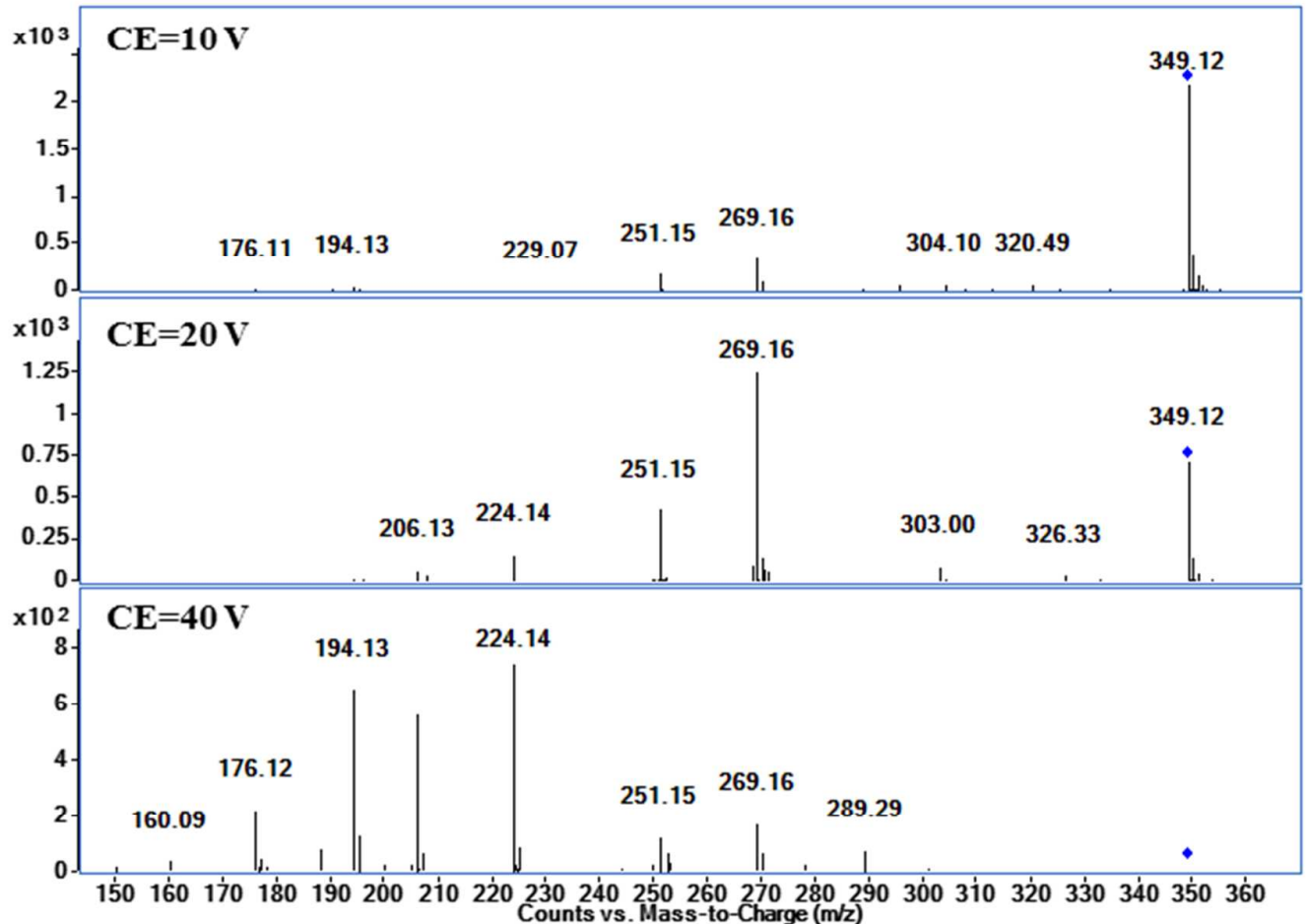




\section{$\mathbf{P}_{349}$}

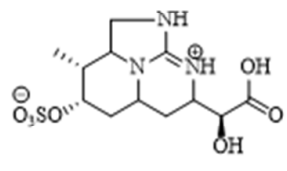

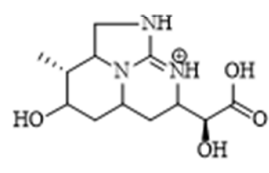

$\mathrm{m} / \mathrm{z}: 270.15$

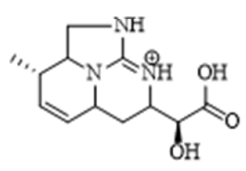

m/2: 252.13

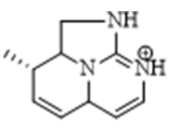

m/z: 194.13

$\mathrm{m} / \mathrm{z}: 176.12$

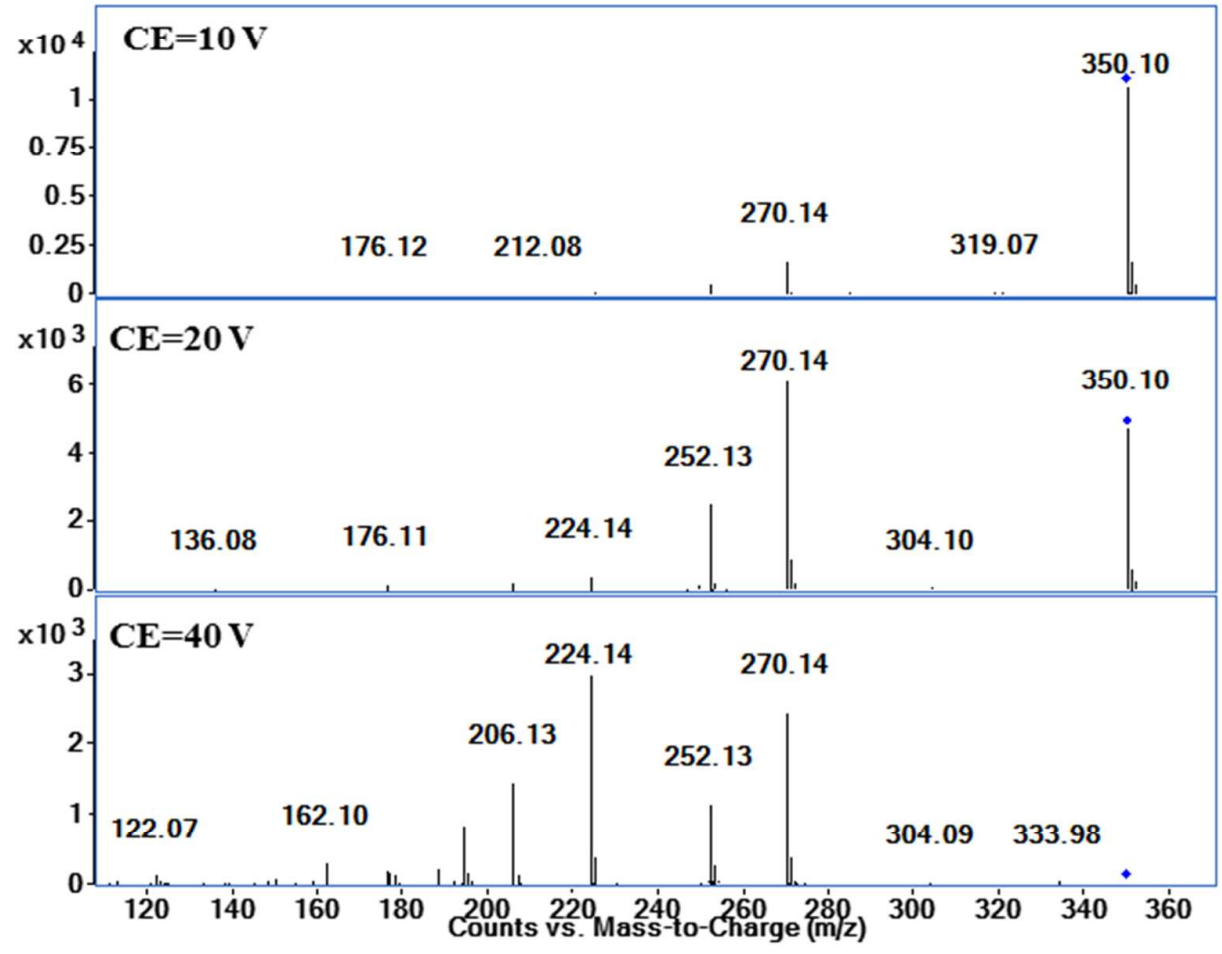




\section{$\mathbf{P}_{319 a}$}

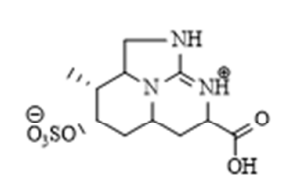

m/z: 320.09

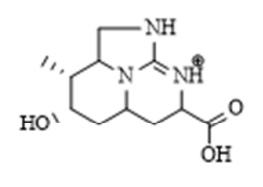

m/z: 240.13

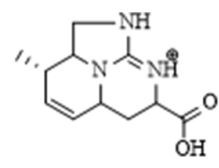

m/z: 222.12
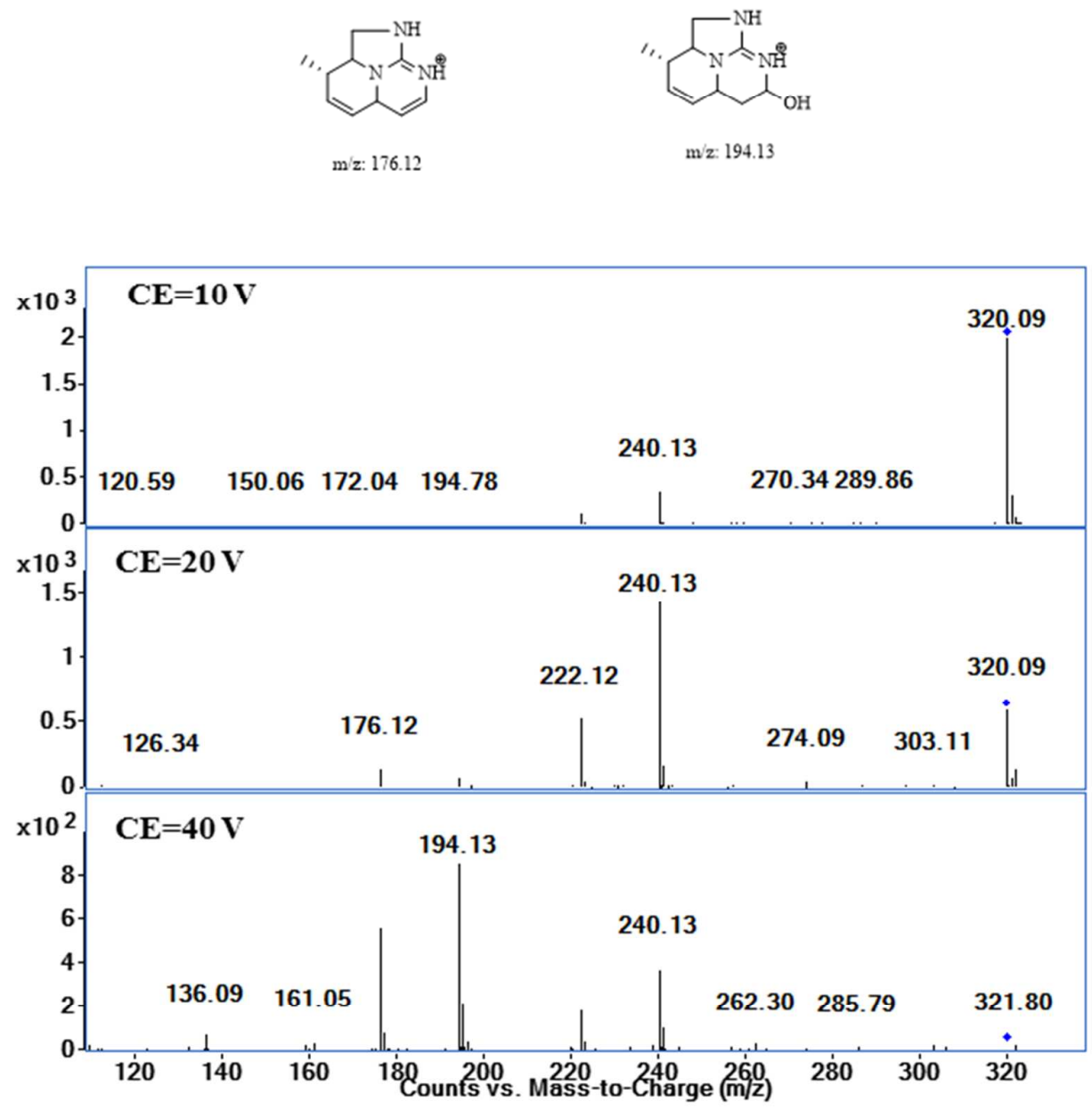


\section{$P_{291 a}$}

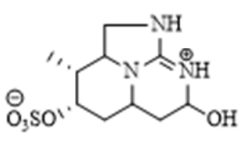

m/z: 292.10

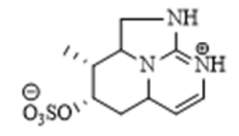

m/z: 274.09

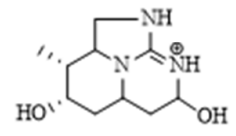

m/z: 212.14

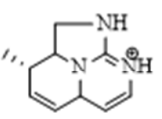

$\mathrm{m} / \mathrm{z}: 176.12$

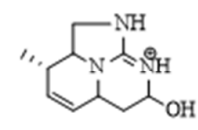

m/z: 194.13

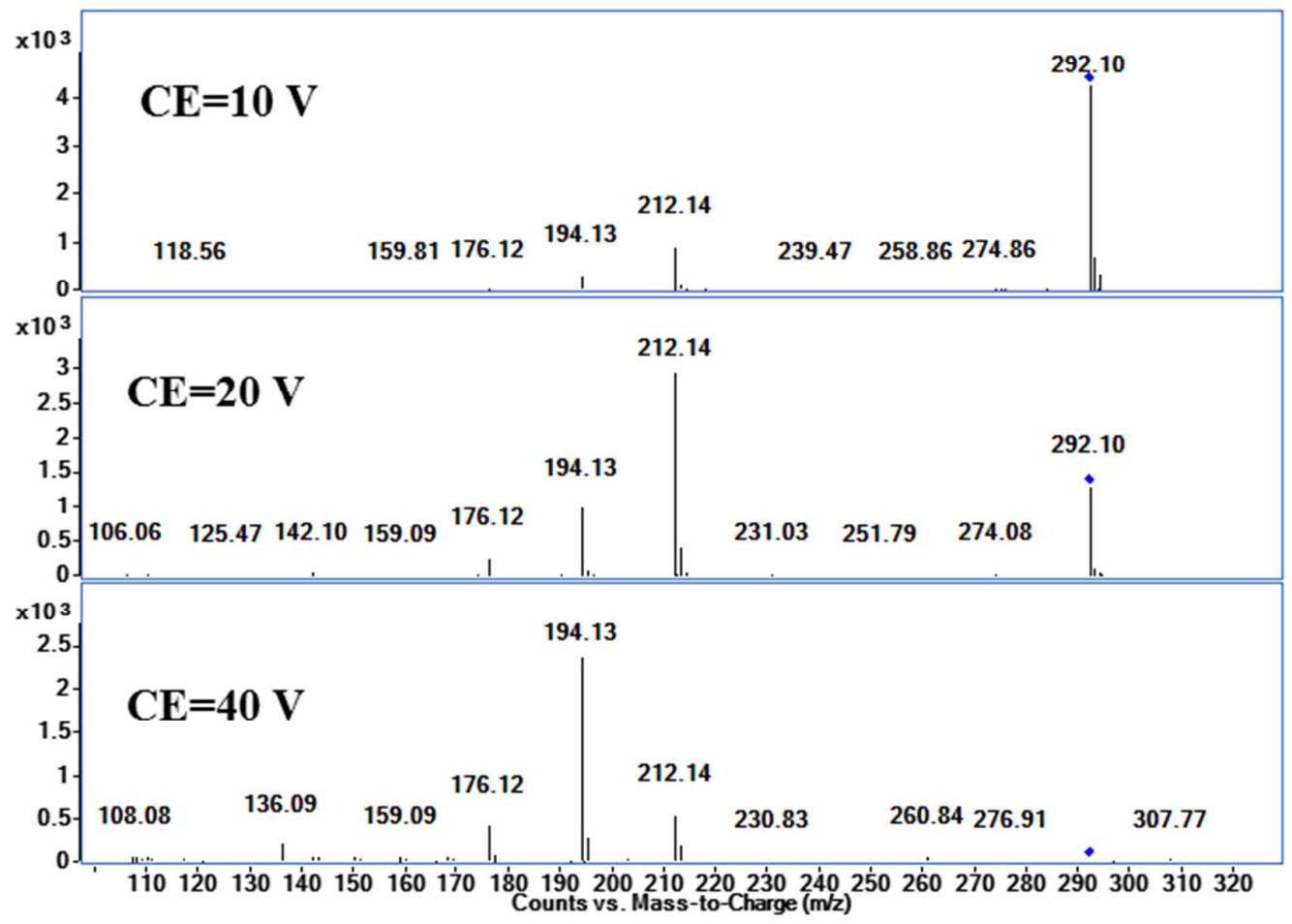




\section{$P_{291 b}$}
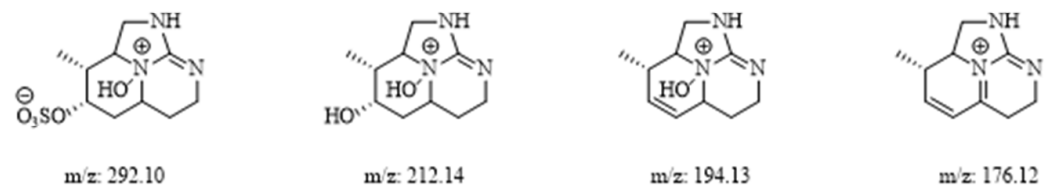

m/z: 194.13

m/z: 176.12

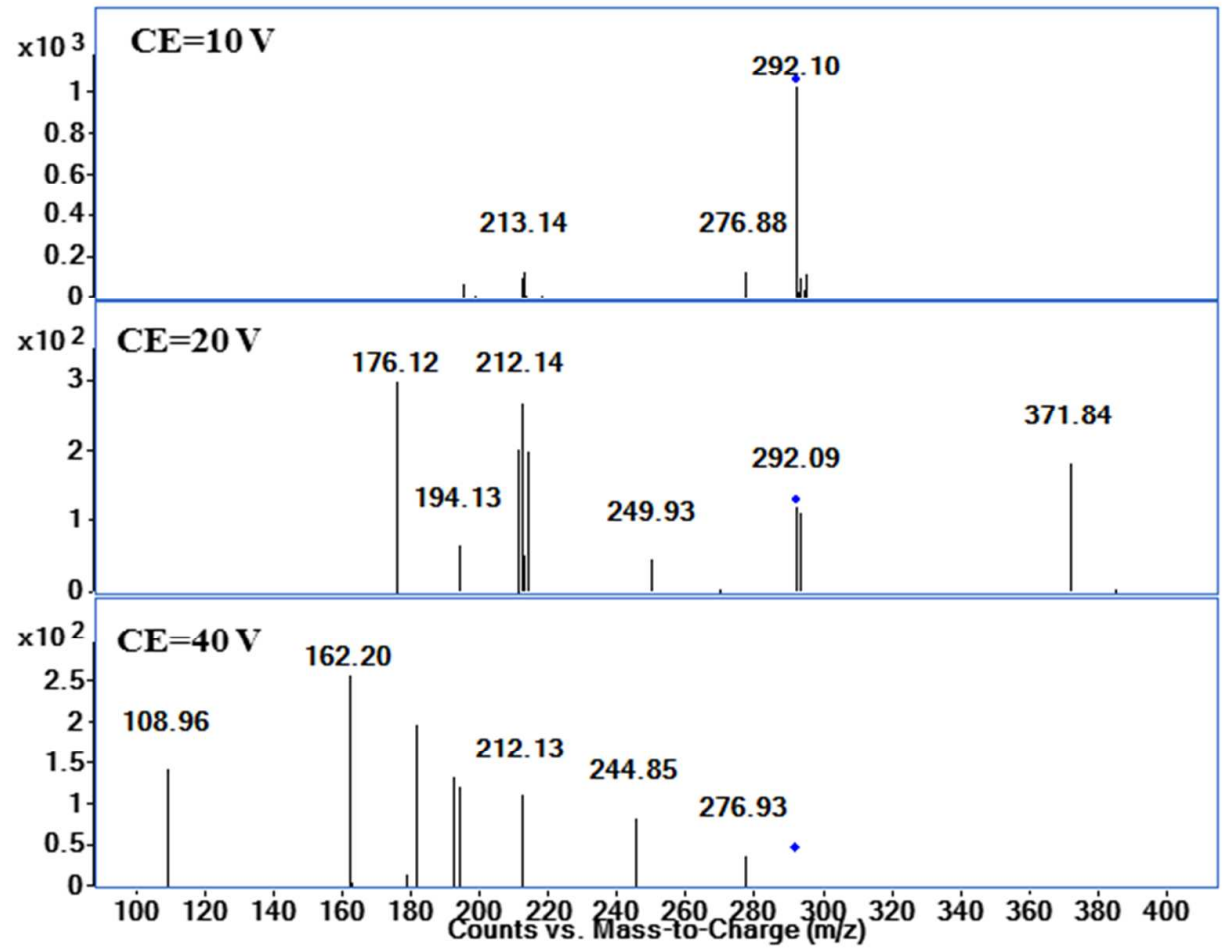




\section{$\mathbf{P}_{365 a}$}

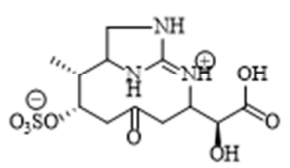

m/z: 366.097

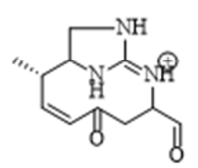

m/z: 222.124

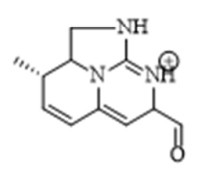

m/z: 204.113

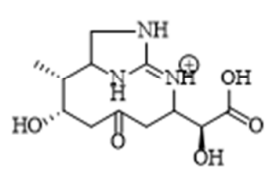

m/z: 286.140

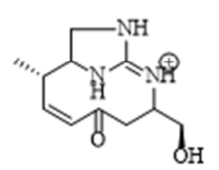

m/z: 224.139

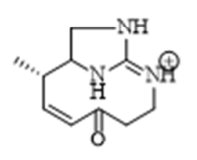

m/z: 194.129

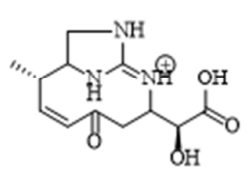

m/z: 268.130

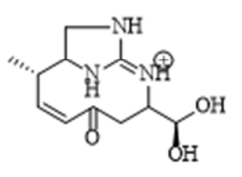

miz: 240.134

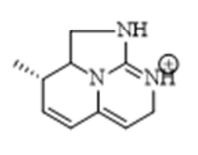

m/z: 176.118

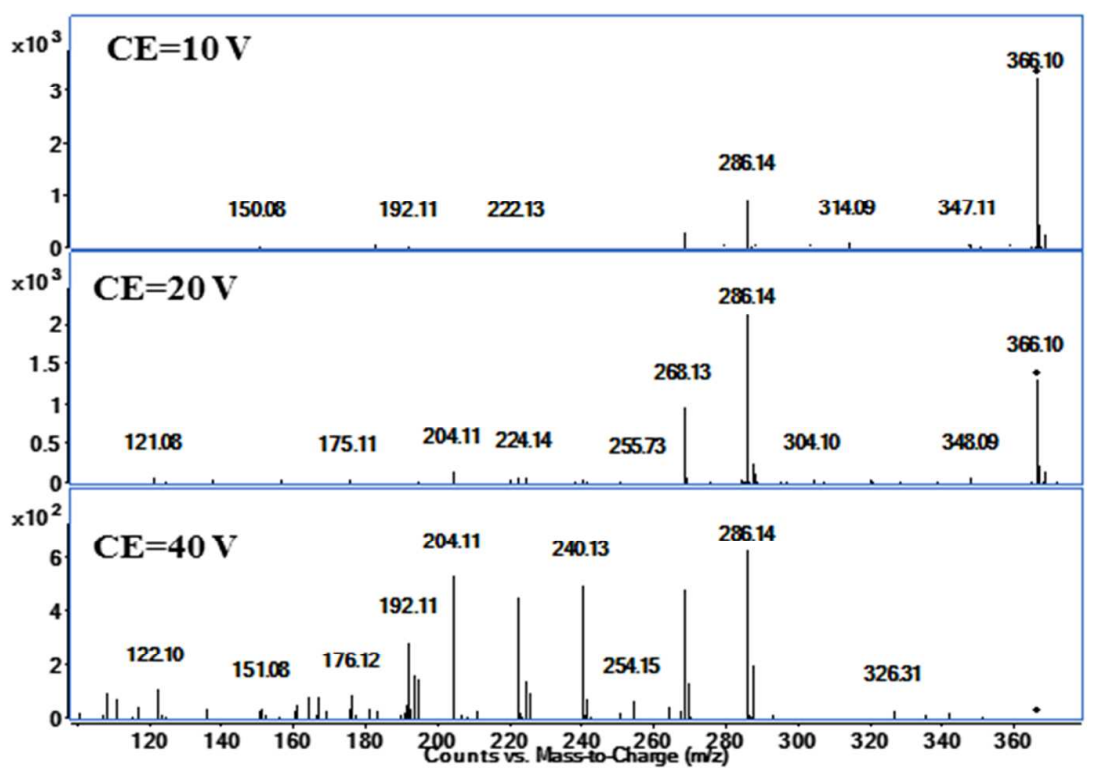




\section{$\mathbf{P}_{390 \mathrm{a}}$}

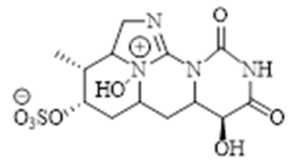

$\mathrm{M} Z \mathrm{Z}=391.1$

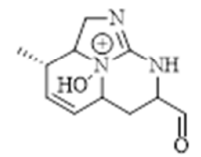

$M / Z=222.124$

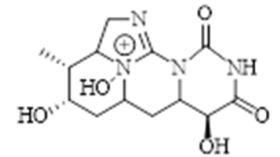

$M / Z=311.14$

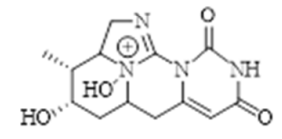

$M / Z=293.124$
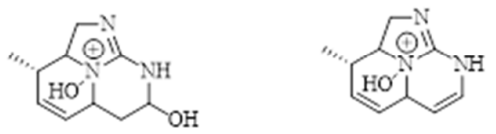

$M / Z=210.124$

$M Z=192.113$

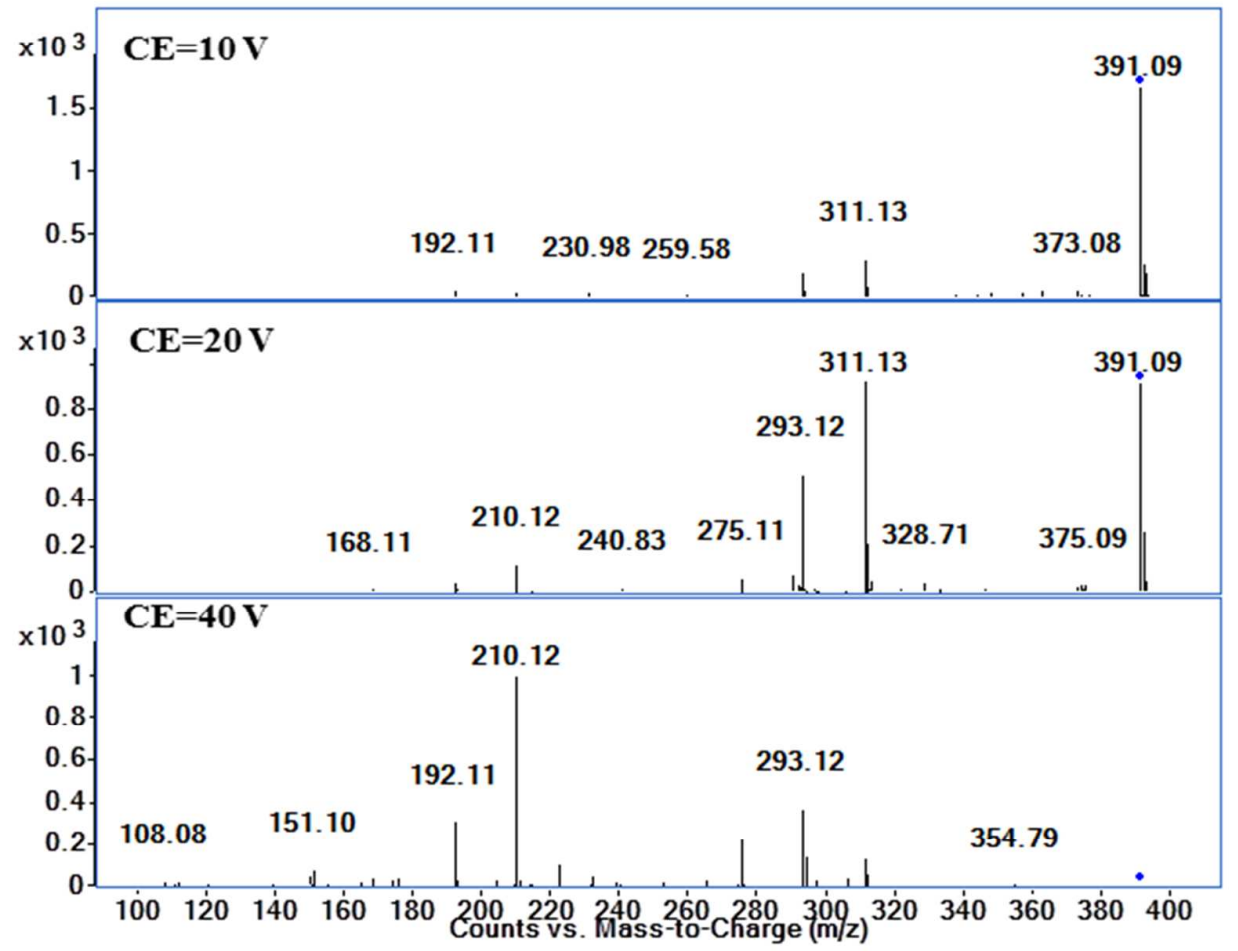




\section{$\mathbf{P}_{390 \mathrm{~b}}$}

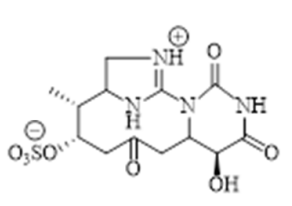

$M / Z=391.1$

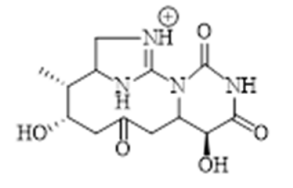

$M / Z=311.135$

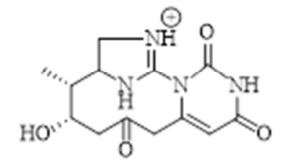

$M Z=293.124$

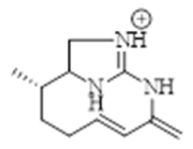

$M / Z=192.15$

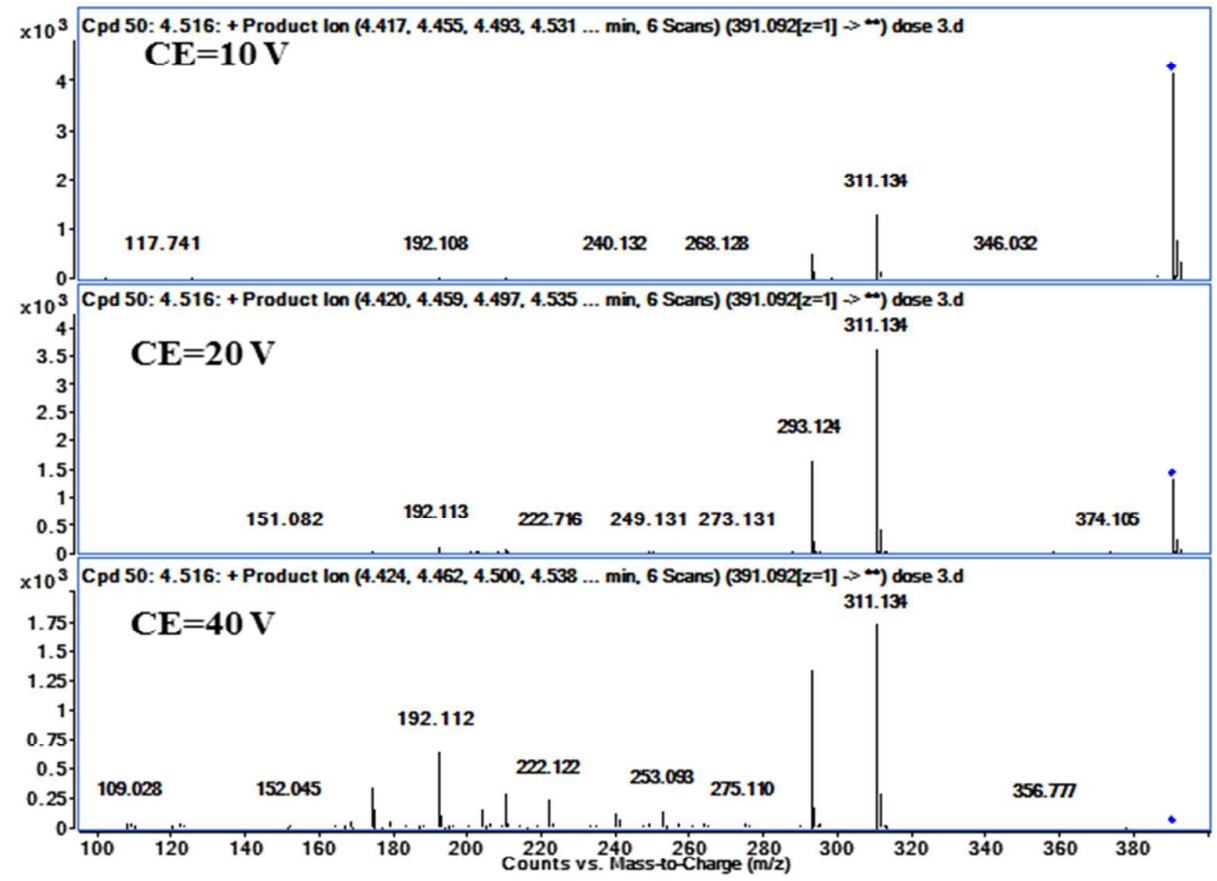




\section{$\mathbf{P}_{321 a}$}
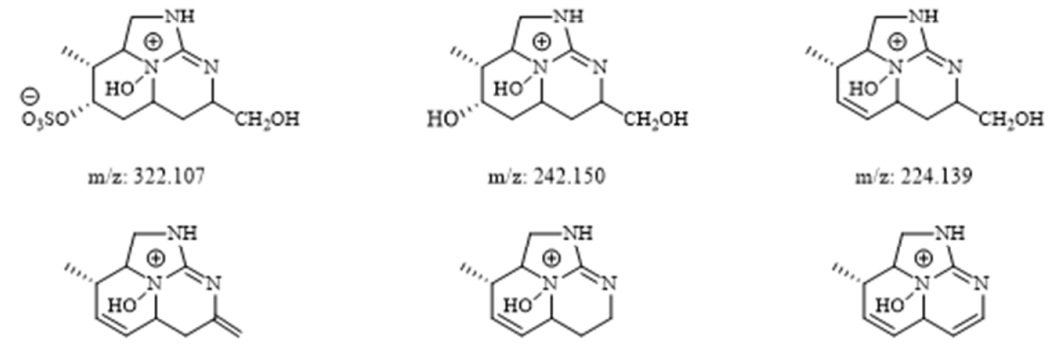

$\mathrm{m} / \mathrm{z}: 242.150$

m/z: 224.139

m/z: 206.129
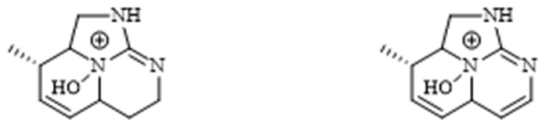

m/z: 194.129

$\mathrm{m} / \mathrm{z}: 192.113$

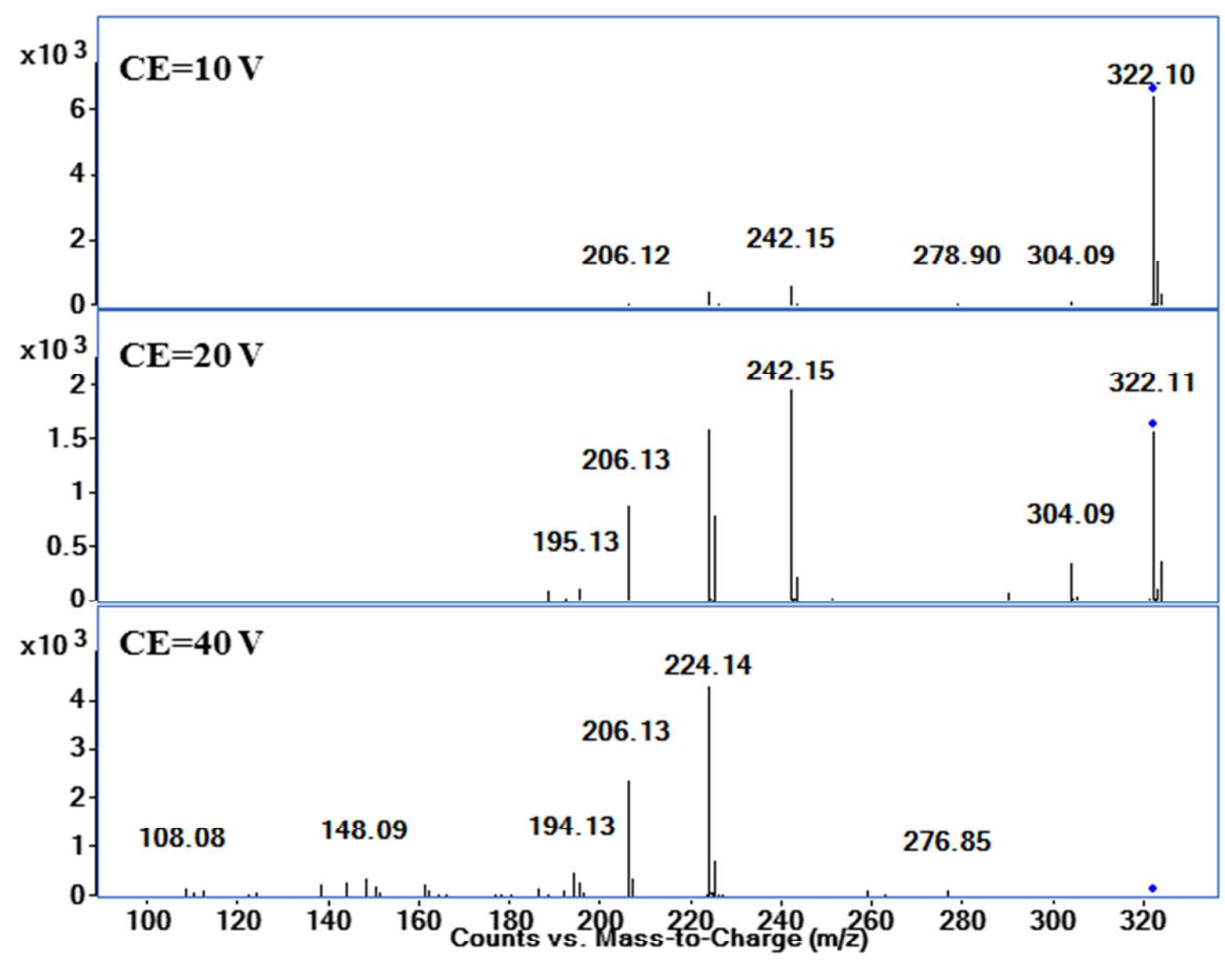




\section{$\mathbf{P}_{321 \mathrm{~b}}$}
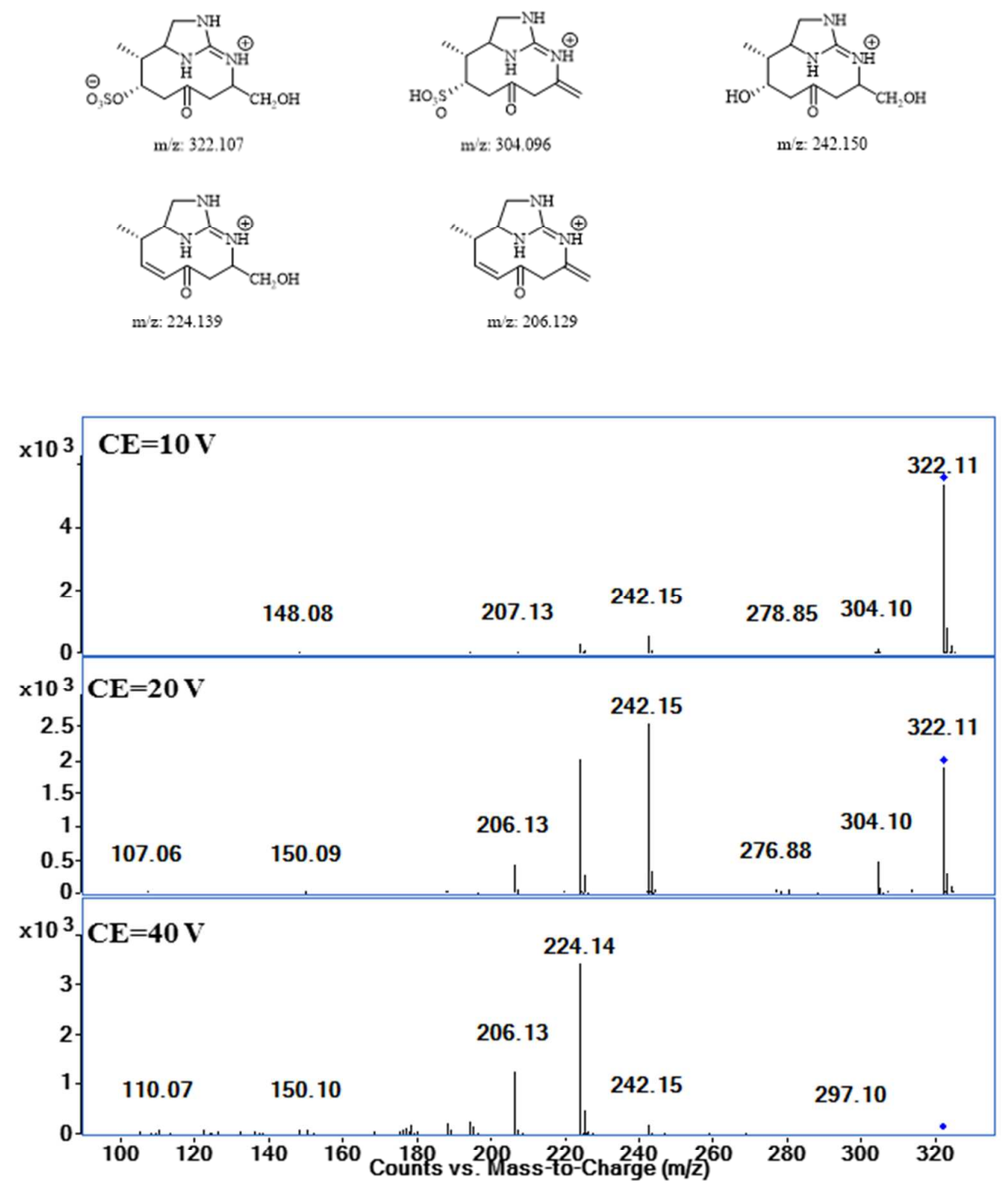


\section{$\mathbf{P}_{337 a}$}

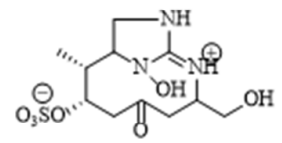

m/z: 338.102

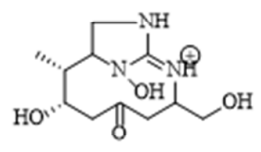

m/z: 258.145

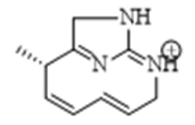

m/z: 176.118

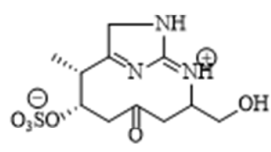

m/z: 320.091

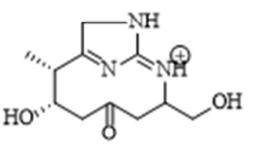

m/z: 240.134

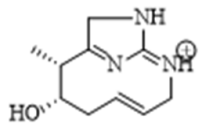

m/z: 194.129

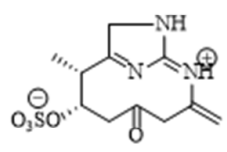

$\mathrm{m} / \mathrm{z}: 302.081$

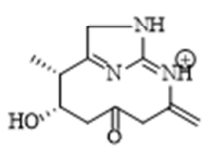

$\mathrm{m} / \mathrm{z}: 222.124$

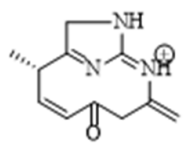

m/z: 204.113

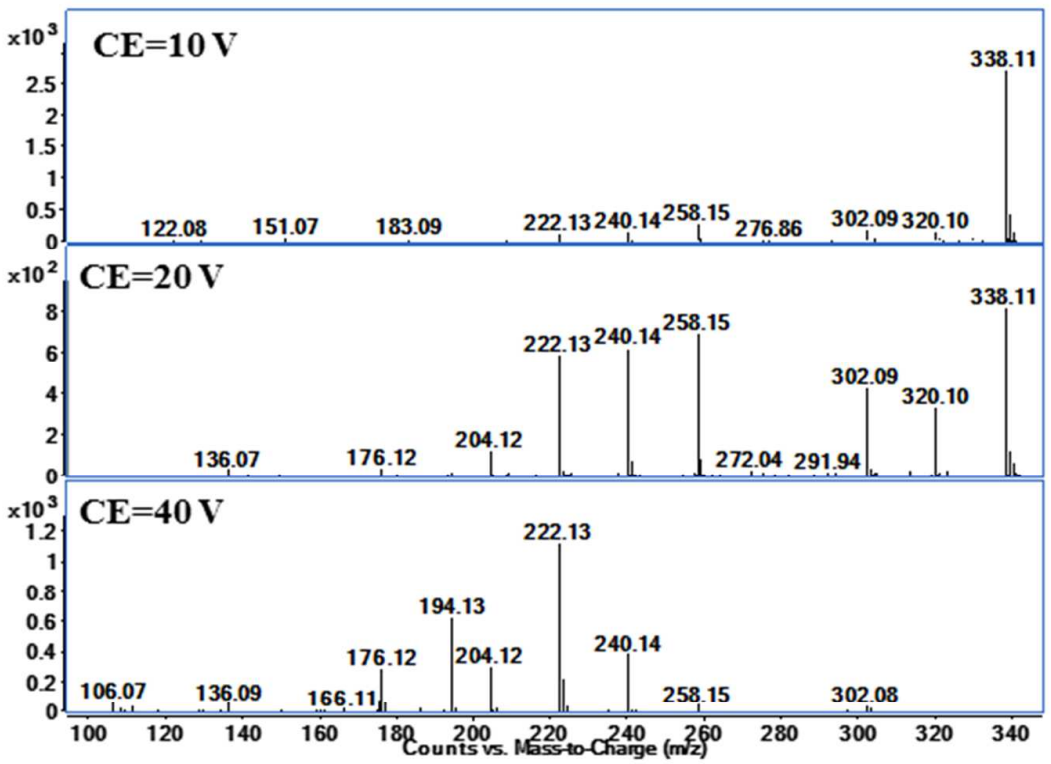




\section{$\mathbf{P}_{337 \mathrm{~b}}$}
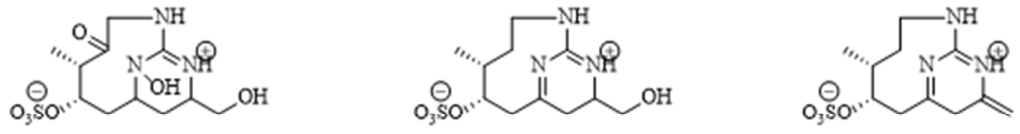

mz: 338.102

m/z: 320.091

m/2: 302.081
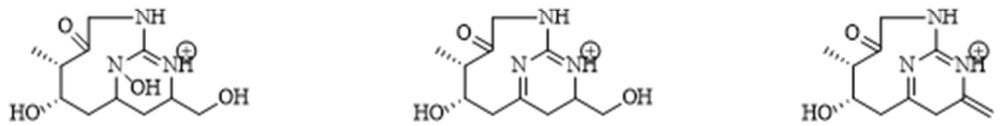

m/z: 258.145

m/z: 240.134

$\mathrm{m} / 2: 222.124$

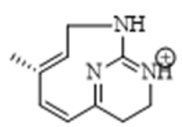

m/z: 176.118

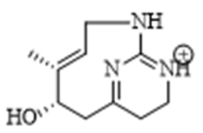

m/z: 194.129

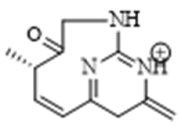

$\mathrm{m} / \mathrm{z}: 204.113$

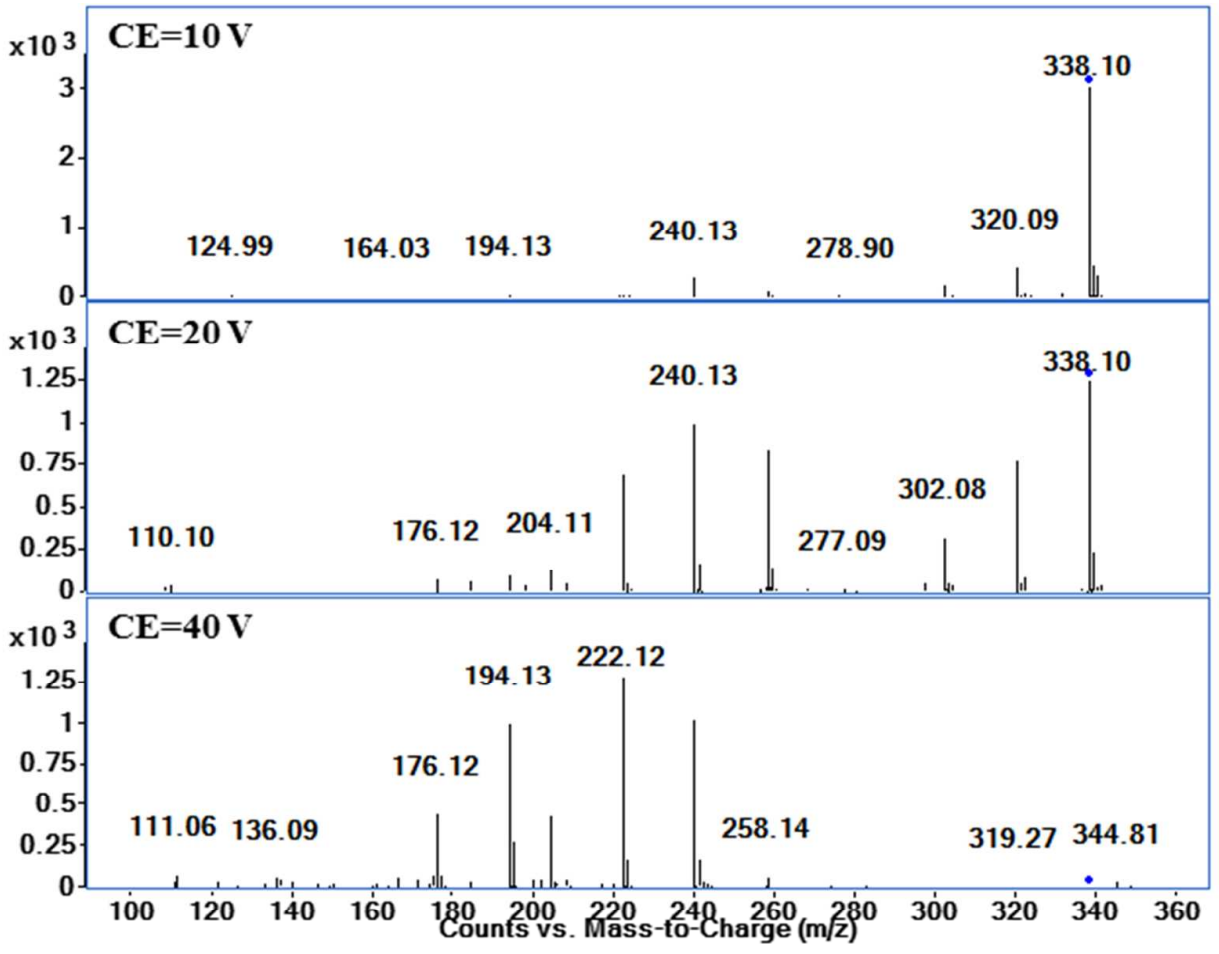




\section{$P_{406 b}$}

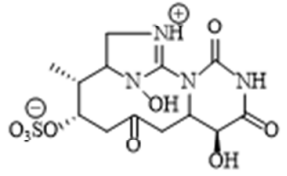

$M / Z=407.09$

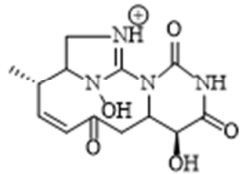

$M / Z=309.119$

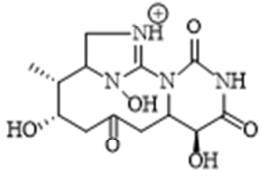

$M / Z=327.130$

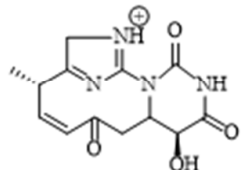

$M / Z=291.109$

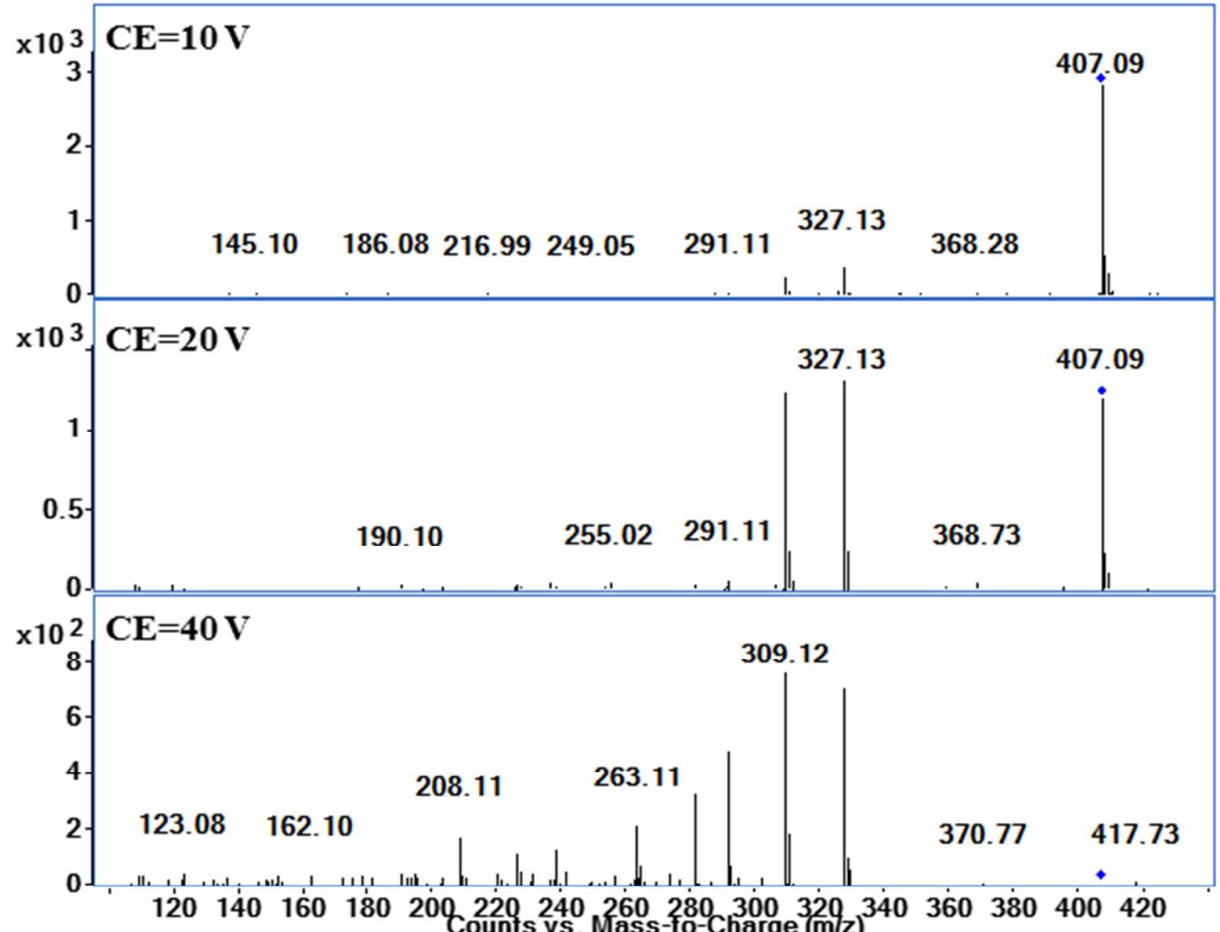




\section{$\mathbf{P}_{335 c}$}

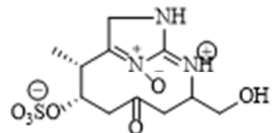

m/z: 336.0806

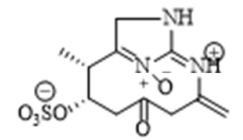

$\mathrm{m} / \mathrm{z}: 318.0754$

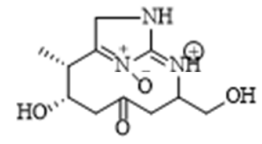

m/z: 256.129?
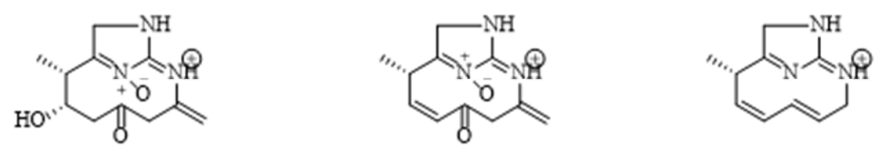

$\mathrm{m} / \mathrm{z}: 238.1186$

$\mathrm{m} / \mathrm{z}: 220.1081$

$\mathrm{m} / \mathrm{z}: 176.1182$

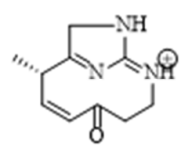

$\mathrm{m} / \mathrm{z}: 192.1131$

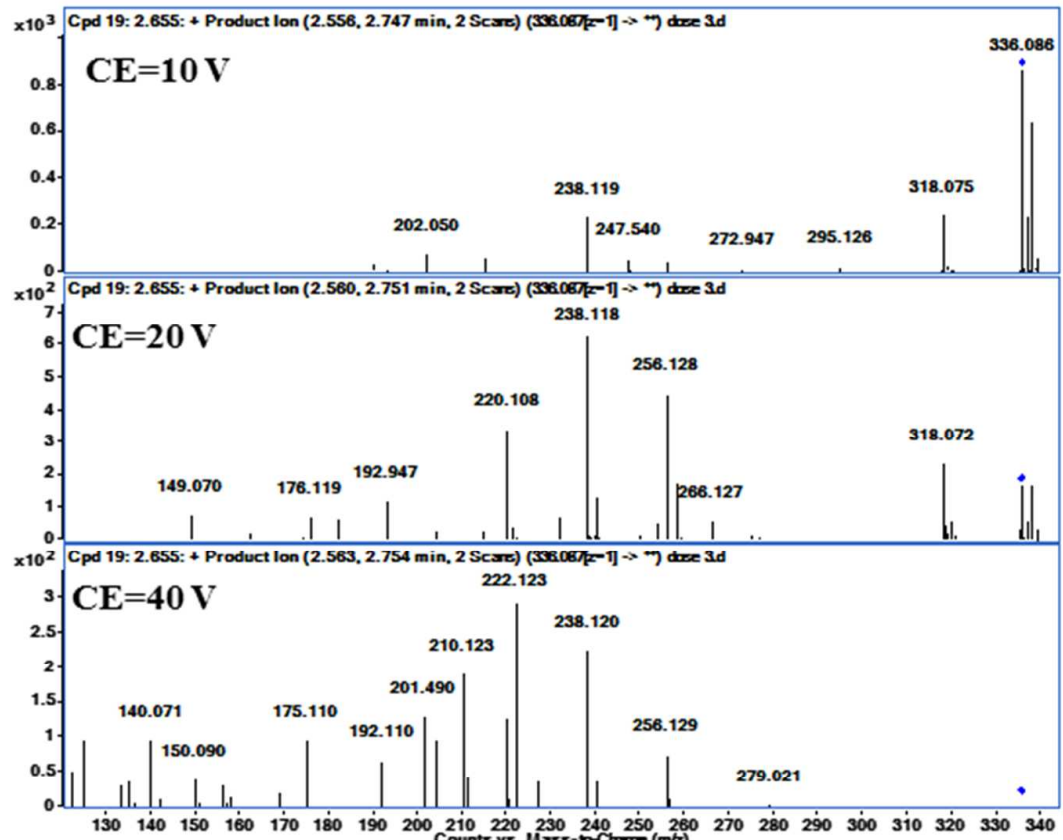




\section{$\mathbf{P}_{404 a}$}

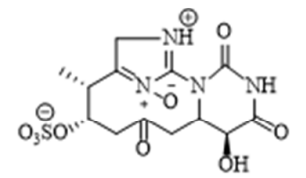

$M / Z=405.071$

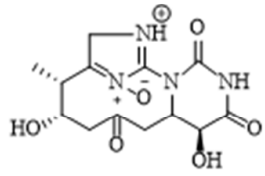

$M / Z=325.114$

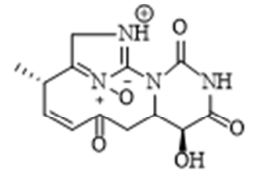

$M / Z=307.104$

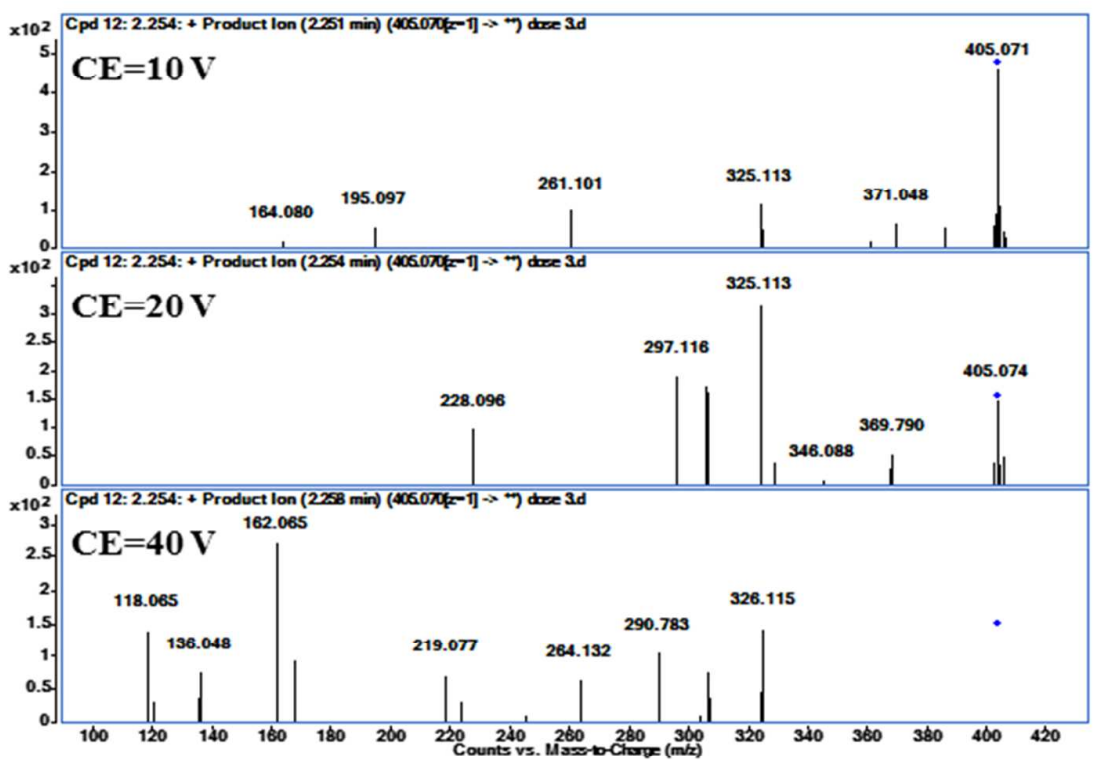




\section{$\mathbf{P}_{305 a}$}
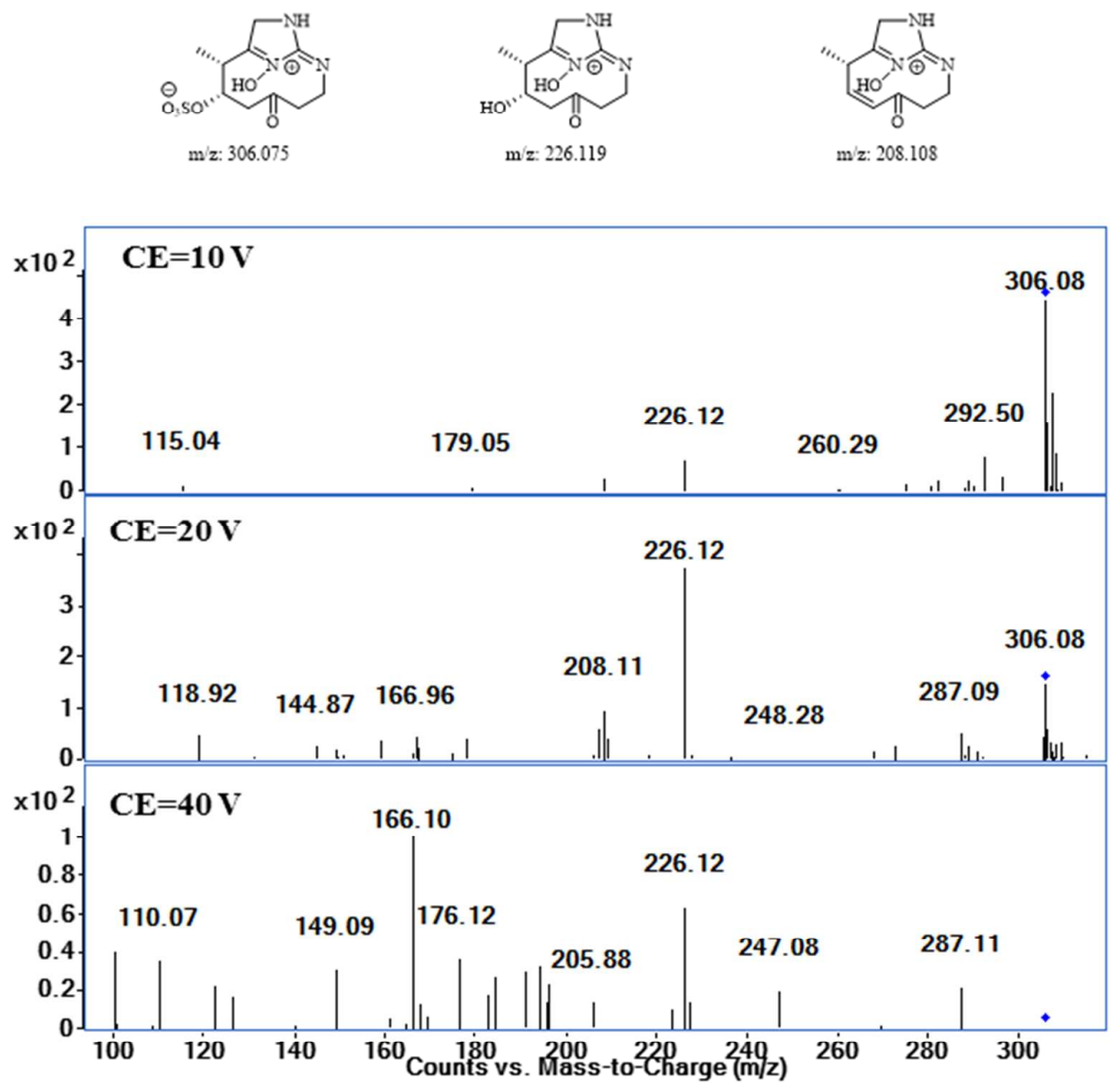


\section{$\mathbf{P}_{305 b}$}
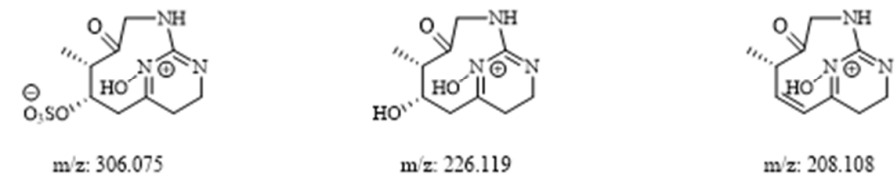

m/z: 208.108

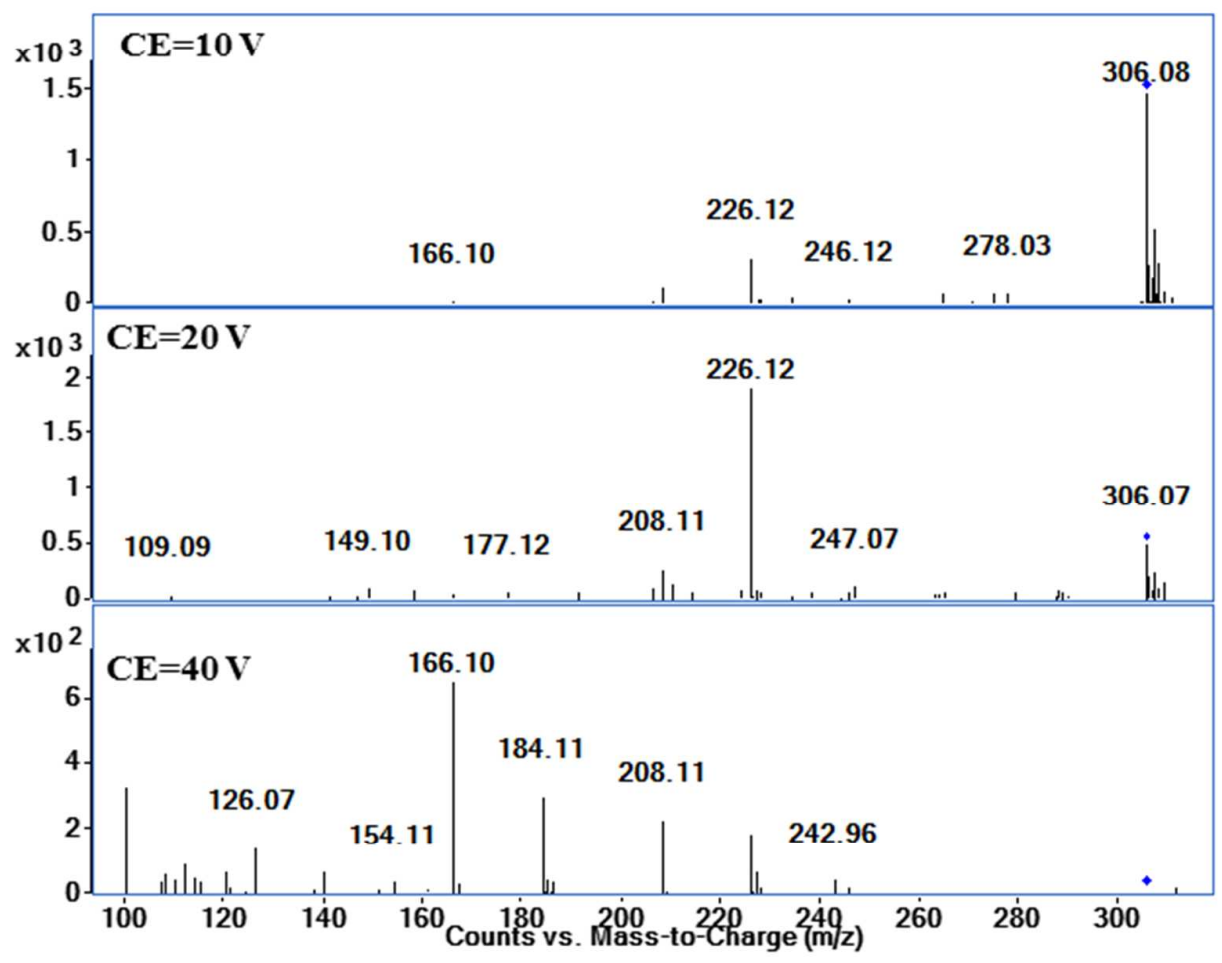

Figure S4. Mass spectrometry fragments of CYN and its identified ozonation products. 

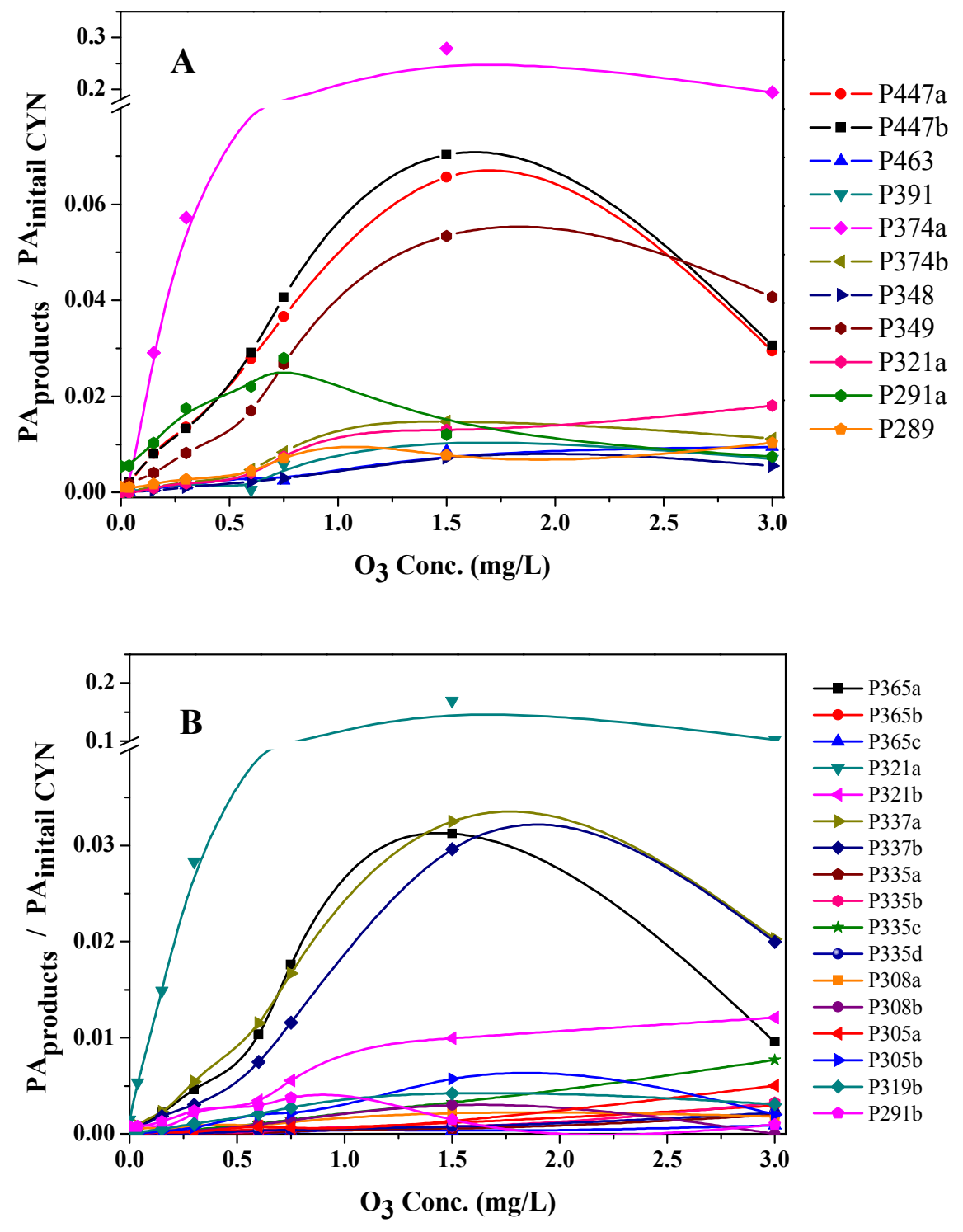


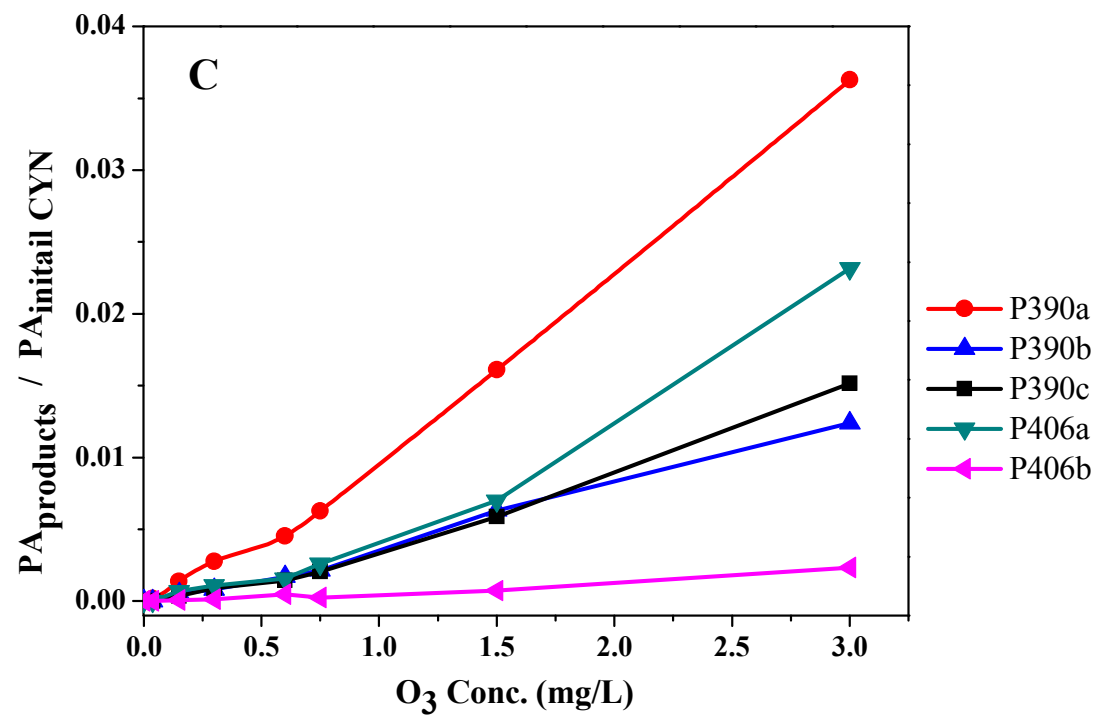

Figure S5. Reaction profile of ozonation products as a function of ozone doses, A: the products generated from the reaction of the uracil moiety. B: the products generated from the reaction of tertiary/secondary amines, start with $\mathrm{P}_{349}(\mathrm{~m} / \mathrm{z} 350.1017), \mathrm{C}$ : the reaction of tertiary/secondary amines, start with $\mathrm{P}_{374 \mathrm{a}}(\mathrm{m} / \mathrm{z}$ 375.0969). "PA" stands for the peak area of the LC-MS results. 

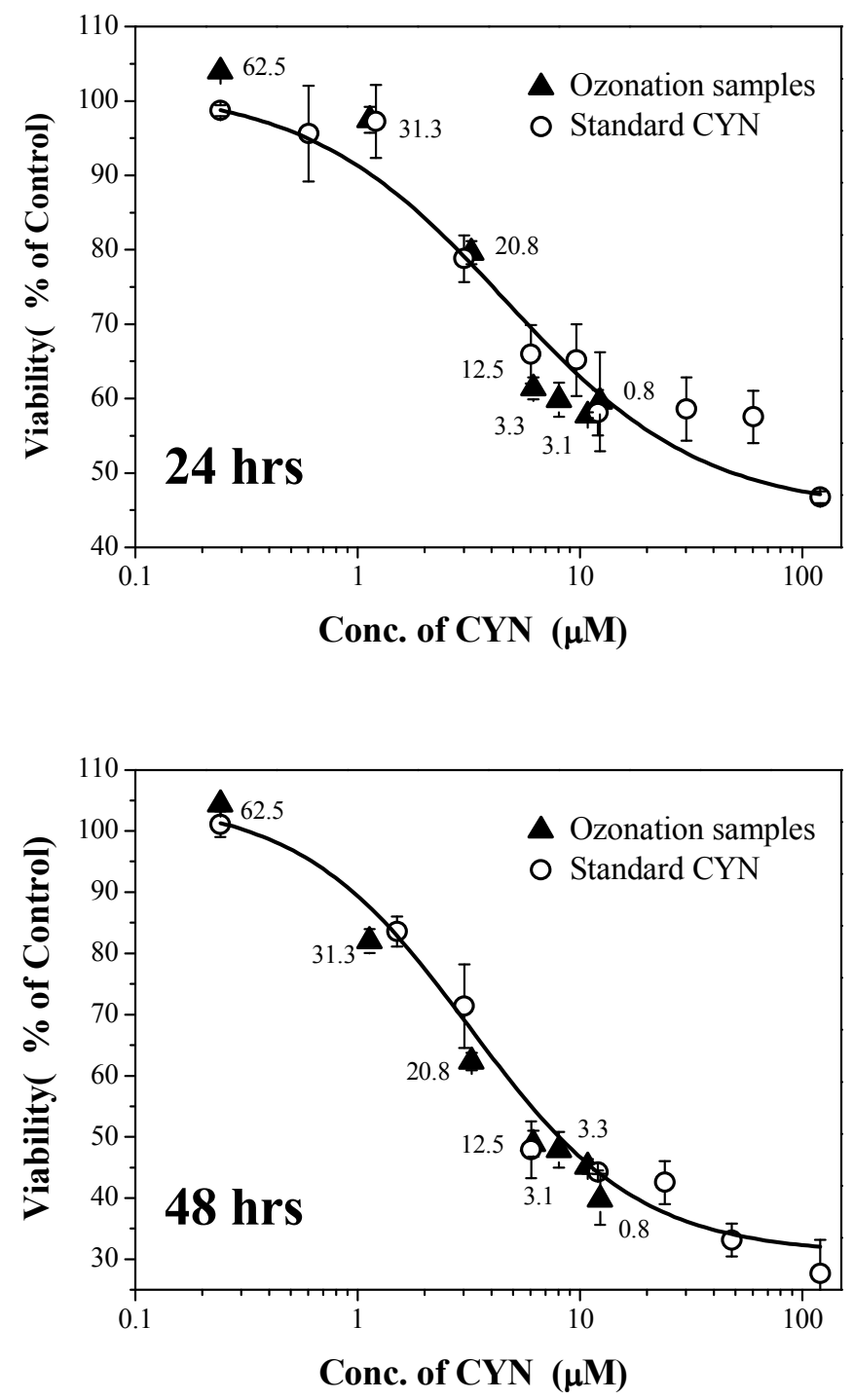

Figure S6. Cytotoxicity results of the CYN for standard inhibition curve and ozonated CYN samples through MTS assay. $\triangle$ stand for CYN for standard inhibition curve. $\boldsymbol{\Delta}$ stand for ozonation of 20.0 $\mu \mathrm{M} C \mathrm{CYN}$ under varied ozone doses (marked as numbers), the concentrations of CYN were measured by LC-TOF. 